\title{
3 Research Square

\section{Ovarian Cancer Cell-Derived Exosomal miR-205 Promotes M2 Macrophage Polarization and Ovarian Cancer Cell Metastasis By Activating The AKT/mTOR Signalling Pathway}

\section{Liuqing He}

Xiangya Hospital Central South University

\section{Quan Chen}

Xiangya Hospital Central South University

\section{Di Wu}

Xiangya Hospital Central South University

Wei Zhu

Xiangya Hospital Central South University

Qifeng Chen

Xiangya Hospital Central South University

Xiaoying Wu ( $\sim$ xyw2007@csu.edu.cn )

Xiangya Hospital Central South University

\section{Research}

Keywords: exosomal, miR-205, Ovarian cancer, Macrophage, Polarization, Metastasis

Posted Date: April 6th, 2021

DOl: https://doi.org/10.21203/rs.3.rs-373729/v1

License: (a) (i) This work is licensed under a Creative Commons Attribution 4.0 International License.

Read Full License 


\section{Abstract}

Background: Tumour-associated macrophages (TAMs) are the most abundant immune cells in the tumour environment and are considered to be similar to M2 macrophages, which facilitate cancer progression. Exosomes, as important mediators of cell-to-cell communication, can alter the phenotype of TAMs by transferring microRNAs (miRNAs) that influence targets and signalling pathways. However, the exact mechanisms by which cancer-derived exosomal miRNAs facilitate the development and metastasis of ovarian cancer (OC) remain unclear.

Methods: In situ hybridization and immunohistochemistry were performed to examine the relationship between miR-205 and CD163 in OC. Exosome labelling experiments and qRT-PCR were used to detect the transfer of miR-205 from OC cells to macrophages. The effects of exosomal miR-205-induced macrophages on OC cell migration, invasion and EMT were assessed by in vitro assays in a co-culture model. Western blotting and qRT-PCR experiments were performed to investigate the role of the $\mathrm{PI} 3 \mathrm{~K} / \mathrm{AKT} / \mathrm{mTOR}$ axis in M2 macrophage polarization induced by exosomal miR-205. An in vivo mouse tumour model was used to evaluate the effects of M2 macrophages induced by exosomal miR-205.

Results: We found that miR-205 expression levels were associated with M2 macrophage infiltration in patients with OC. miR-205 could be transported from OC cells to macrophages via exosomes and altered the macrophage phenotype. Moreover, macrophages that received exosomal miR-205 further enhanced the invasion, migration and EMT of OC cells. Decreased PTEN levels caused by exosomal miR-205 could increase the activation of AKT and mTOR as well as the expression of several immunosuppressive factors. In contrast, inhibition of miR-205 or restoration of PTEN effectively decreased cancer-mediated M2-type polarization, improving the infiltration of inflammatory factors in the tumour environment. Exosomal miR-205 derived from OC cells was found to induce M2-type polarization of macrophages and promote cancer progression in vivo.

Conclusions: These results suggest a novel mechanism by which exosomal miR-205 induces M2 macrophage polarization and facilitates OC progression by targeting the PI3K/AKT/mTOR axis. Targeting exosomal miR-205 may offer a potential diagnosis and treatment strategy for OC.

\section{Background}

Ovarian cancer $(\mathrm{OC})$ is the most lethal malignant tumour of the gynaecological reproductive system, and its high mortality rate is related to its high frequency of invasion and early metastasis [1]. Generally, high rates of metastasis in $\mathrm{OC}$ are related to poor survival rates, but the mechanisms of invasion and metastasis in $\mathrm{OC}$ are still unclear, and effective early screening measures to diagnose and prevent the metastasis of $\mathrm{OC}$ are still lacking. Therefore, finding new diagnostic biomarkers and revealing the molecular mechanisms underlying the metastasis of $\mathrm{OC}$ are significant research challenges.

Tumour-associated macrophages (TAMs) represent a prominent immune population in the tumour microenvironment (TME) and play significant roles in various processes, including tumour cell migration, 
invasion and premetastatic site formation during cancer progression [2-6]. In the TME, macrophages can be either tumour-promoting or tumour-suppressing, depending on their functional polarization. Classically (M1) and alternatively (M2) activated macrophages can display diverse phenotypes and functions depending on environmental cues $[7,8]$. M1 macrophages, representing a pro-inflammatory phenotype, are stimulated by the Th1 cytokine interferon (IFN)- $\gamma$ and express pro-inflammatory factors such as interleukin (IL)-6, IL-1 $\beta$ and tumor necrosis factor (TNF)-a to destroy cancer cells [9]. Conversely, M2 macrophages, considered to be the main phenotype of TAMs, express high levels of macrophage mannose receptors (CD206) and CD163, as well as anti-inflammatory cytokines IL10 and IL8, to favour tumour cell growth [10]. Cross-talk between tumour cells and macrophages is crucial to TME formation and cancer progression [11-13]. However, the underlying mechanisms of TAMs promoting cancer progression and the interaction between cancer cells and macrophages remain largely unknown.

A growing body of studies has shown that cell-cell communication can be initiated through exosomes [14-16]. Exosomes are extracellular vesicles released from various cell types and contain numerous molecules from the parental cells, including microRNAs (miRNAs), proteins and nucleic acids [17-19]. The transfer of miRNAs by exosomes is especially interesting, since exosomal miRNAs released from cancer cells could stimulate macrophages to switch their phenotype and role in promoting cancer progression and metastasis [20-22].

In this study, we found that high expression of miR-205 was associated with M2 macrophage infiltration in OC patients. M2 macrophages affected OC cell invasion, migration and EMT via exosomal miR-205. Moreover, OC cell-derived exosomal miR-205 could polarize macrophages to the M2 type via the $\mathrm{PTEN} / A K T / m T O R$ signalling pathway. The polarization of M2 macrophages by exosomal miR-205 led to enhanced OC development and metastasis in vivo. We conclude that miR-205 upregulation in OC alters the macrophage phenotype towards protumour activity through exosome-mediated delivery of oncogenic miR-205. Thus, the data indicate that miR-205 may serve as a target for exosome-mediated tumour immune evasion.

\section{Methods}

\section{Human tissue collection}

The 74 tissues used in this study were collected from patients with primary OC who were treated at Xiangya Hospital of Central South University (CSU) between 2014 and 2018. All tumour tissues were obtained at the time of primary surgery, and patients were deemed optimally debulked. Among the 74 tissues, 20 normal ovarian tissues were collected from the contralateral normal ovaries of OC patients, 20 metastatic tissues were obtained from distant metastases of OC patients, and $34 \mathrm{OC}$ samples were obtained from primary tumour lesions of OC patients. The study was approved by the Institutional Ethics Committee of Xiangya Hospital of CSU and was conducted following the ethical guidelines of the Declaration of Helsinki. Each participant signed an informed consent form.

\section{Cell culture}


The epithelial OC cell lines HO-8910, HO-8910PM, SKOV-3, SKOV-3ip, and OVCAR-3 and the monocytic cell line THP-1 were obtained from the Chinese Academy of Sciences Cell Bank and had been purchased from China Center for Type Culture Collection (CCTCC) and Cell Resource Center, IBMS, CAMS/PUMC (CRC/PUMC). HO-8910 cells stably overexpressing miR-205 and negative control cells were established by the Xiaoying Wu research group of the pathology laboratory of CSU, and the transfection efficiency was verified in a previous study [23]. All cell lines were cultured in DMEM or RPMI 1640 medium (Thermo Scientific, Waltham, MA, USA) supplemented with $10 \%$ foetal bovine serum (FBS) (Gibco, Gaithersburg, MD, USA). THP-1 cells $\left(1 \times 10^{6}\right)$ were incubated with $100 \mathrm{ng} / \mathrm{ml}$ phorbol 12-myristate 13-acetate (PMA) (Solarbio, Beijing, China) for 24-48 $\mathrm{h}$ in vitro to induce their differentiation into macrophages. For the exosome treatment experiments, $1 \mu \mathrm{g} / \mathrm{ml}$ exosomes was added to the culture medium of recipient cells (2 $\left.\times 10^{5}\right)$.

\section{Immunohistochemistry (IHC) and in situ hybridization (ISH)}

A total of 74 paraffin-embedded OC tissues were assessed by IHC and ISH staining. CD68 and CD163 expression was measured with anti-CD68 (1:200, Abcam, Cambridge, UK) and anti-CD163 (1:200, Abcam) antibodies. ISH was performed on OC tissue samples using an LNA microRNA ISH miR-205 optimization kit (Exiqon; Woburn, MA, USA). The experimental steps of IHC and ISH were the same as in our previous study [24].

\section{Exosome isolation and characterization}

The cell lines were cultured in normal medium until $60 \%-70 \%$ confluence, thereafter, the medium was replaced with fresh medium supplemented with $10 \%$ exosome-depleted FBS. Then, the cell culture medium was harvested after $48 \mathrm{~h}$ and centrifuged (Beckman Coulter, Brea, CA, USA) at 2,000×g for 30 min at $4^{\circ} \mathrm{C}$ to remove residual cells, debris and remaining macropolymers. The supernatant was passed through a $0.22-\mu \mathrm{m}$ filter (Millipore, Danvers, MA, USA). After centrifugation at 3,000 $\times \mathrm{g}$ for $30 \mathrm{~min}$ at $4^{\circ} \mathrm{C}$ in a dialysis tube (Millipore), the supernatant was incubated with ExoQuick-TC ${ }^{\mathrm{TM}}$ exosome precipitation solution (System Biosciences, Palo Alto, CA, USA) for $6 \mathrm{~h}$ to overnight at $4^{\circ} \mathrm{C}$. Subsequently, the mixture was centrifuged at $1,500 \times \mathrm{g}$ for $30 \mathrm{~min}$ at $4^{\circ} \mathrm{C}$ to harvest the yellow exosome pellets. The exosome pellets were resuspended in $100 \mu \mathrm{l}$ phosphate-buffered saline (PBS) for further assays. Western blotting analysis was used to characterize the extracellular vesicle-associated protein markers Hsp70 (Abcam) and CD63 (Abcam) and the exosome-specific marker TSG101 (Abcam). For transmission electron microscopy (TEM), the exosomes were fixed with 1\% glutaraldehyde, dropped in 300-mesh carbon/formvar-coated grids, stained with $2 \%$ uranyl acetate, dried and imaged by transmission electron microscopy (TEM) (FEl, Hillsboro, OR, USA) , as previously reported [25]. The amount of exosomes was measured using the bicinchoninic acid (BCA) protein assay kit (Novagen, Merck Group, Madison, USA).

\section{Exosome labelling and tracking}

To monitor exosome trafficking, purified exosomes isolated from the culture medium were collected and labelled with PKH67 red fluorescent membrane linker dye (Sigma-Aldrich, Merck KGaA) according to the 
manufacturer's instructions. Next, the PKH26-labelled exosomes were washed and centrifuged at $100,000 \times \mathrm{g}$ for $20 \mathrm{~min}$ at $4^{\circ} \mathrm{C}$ to collect the exosomes, which were resuspended and added to unstained macrophages for exosome uptake studies. After treatment with $\mathrm{PKH} 26$-labelled exosomes for $4 \mathrm{~h}$ at $37^{\circ} \mathrm{C}$, the cells were detected by fluorescence microscopy.

\section{RNA interference, vector transfection and qRT-PCR}

The miR-205 mimic (10 nM), inhibitor (20 nM), negative control (10 nM) and PTEN expression plasmid (2 $\mu \mathrm{g}$, GeneCopoeia) were transfected into cells using Lipofectamine 2000 reagent (Invitrogen) according to the manufacturer's protocol. The cells were collected for further assays at 24 or $48 \mathrm{~h}$ after transfection. The transfection efficiencies were verified by fluorescence microscopy and RT-PCR analysis. Total RNA from cells and exosomes was extracted using TRIzol reagent (Invitrogen), and complementary DNA was synthesized with a reverse transcription system (GeneCopoeia, Guangzhou, China) according to the manufacturer's instructions. Reverse transcription and qRT-PCR were performed as previously described [26]. The relative expression levels were evaluated using the $\Delta \Delta C t$ method. The $2^{-\Delta \Delta C t}$ method was applied to analyse the results. The primers for miR-205 mimic, miR-205 inhibitor, miR-205 nc, CD163, CD68, IL10, CCL8, IL-1 $\beta$, TNF-a and PTEN (GeneCopoeia) are available in the Supplementary Table 1.

\section{Western blot analysis}

Western blot analysis was performed as previously described [27]. Proteins extracted from cells or exosomes were lysed in RIPA lysis buffer supplemented with protease inhibitor and quantified using a BCA protein assay kit (Novagen, Merck Group, Madison, USA). Approximately $30 \mu \mathrm{g}$ of protein lysates (from each sample) were separated on 10\% SDS-PAGE gels and transferred onto polyvinylidene difluoride membranes (Millipore). The membranes were blocked with $5 \%$ blocking buffer overnight at $4^{\circ} \mathrm{C}$ with primary antibodies, followed by incubation with a secondary antibody at room temperature. The primary antibodies included anti-CD63, anti-TSG101, anti-Hsp70, anti-E-cadherin, anti-vimentin, anti-PTEN, antimTOR, anti-p-mTOR, anti-AKT, anti-p-AKT, anti-4EBP1, anti-p-4EBP1 (Cell Signaling Technology, USA) and anti-a-tubulin (Proteintech, Chicago, USA). The chemiluminescence signal was detected using an enhanced chemiluminescence mixture (Sigma-Aldrich), and images were captured using a gel imaging system (Bio-Rad Laboratories, CA, USA).

\section{Co-culture, cell migration and invasion assays}

Before starting the assays, THP-1 cells $\left(1 \times 10^{4}\right)$ were seeded on the bottom chamber of 8-mm Transwell inserts (Corning, Sigma-Aldrich) with PMA $(100 \mathrm{ng} / \mathrm{ml})$ for $48 \mathrm{~h}$. Then, the THP-1 cells were differentiated into M0 macrophages, and the supernatant was replaced with normal medium containing $10 \%$ FBS for $24 \mathrm{~h}$ to eliminate the effect of PMA. Tumour cells $\left(5 \times 10^{5}\right)$ were seeded on the upper side of the co-culture system before the co-culture assay. After treatment with miR-205-Exos or miR-205 mimic/NC/inhibitor (RiboBio, Guangzhou, China), M0 macrophages were co-cultured with HO-8910 cells. Cell migration and invasion assays were performed as previously described [23]. 


\section{Animal experiments}

All animal experiments were performed in accordance with the National Institutes of Health $(\mathrm{NIH})$ Guidelines for the Care and Use of Laboratory Animals and according to the protocols approved by the Animal Care and Use Committee at CSU. Female BALB/c nude mice (4-5 weeks old, 18 20 g) were housed in a specific pathogen-free environment in the Animal Laboratory Unit of CSU and were randomly divided into two groups ( $n=6 /$ group). HO-8910 cells $\left(1.5 \times 10^{6}\right.$ cells) mixed with conditioned macrophages pretreated with NC-Exos or miR-205-Exos were injected intraperitoneally (i.p.) into different groups. The procedure of living animal bioluminescence imaging was described previously [24].

\section{Statistical analysis}

All experiments were performed in triplicate. Data are presented as the mean \pm standard error of the mean (SEM). Differences between treated and control groups were analysed using Student's $t$ test or one-way ANOVA. Survival curves were estimated using the Kaplan-Meier method, and the log-rank test was used to calculate differences between the curves. A P-value less than 0.05 was considered statistically significant. Statistical analyses were performed by GraphPad Prism 5.0 (GraphPad Software, Inc., La Jolla, CA, USA).

\section{Results}

\section{The expression levels of miR-205 correlate with macrophage infiltration in patients with OC}

M2 macrophages, which are believed to be the main phenotype of TAMs, promote the progression and metastasis of tumours. In this study, an anti-CD163 antibody was first used to identify M2 macrophages by IHC. The expression level of CD163 was upregulated in both primary and metastatic tumour sites compared with normal ovarian tissue (Fig. 1a and b). In addition, the association of CD163 expression and the prognosis of $\mathrm{OC}$ patients was assessed by using the Kaplan-Meier plotter database, which showed that patients with high CD163 expression had significantly shorter overall survival times than OC patients with low CD163 expression (Fig. 1c and d). Previous studies have found that the upregulation of miR-205 is associated with poor prognosis in OC patients. Importantly, we found that CD163 expression levels were higher in OC specimens with high miR-205 expression than in those with low miR-205 expression (Fig. 1e). Moreover, Spearman correlation analysis suggested that there was a corresponding upregulation of miR-205 when CD163 was increased (Fig. 1f). These results suggest that miR-205 expression is associated with TAM infiltration. Interestingly, we found that miR-205 expression appeared to predominantly involve tumour-associated immune cells and tumour cells (Fig. 1g). Based on their morphology as well as reports from other cancer types [11], we reasoned that the majority of miR-205positive immune cells observed in OC might be TAMs. To test this hypothesis, ISH and IHC were performed to detect miR-205, the macrophage marker CD68 and the M2-type macrophage marker CD163 in the same independent sets of OC specimens. The following staining patterns were observed: miR-205 staining on both tumour cells and CD68+ and CD163+ cells in most of the OC tissue samples (31/54, Fig. 
1h). Therefore, according to these results, we hypothesized that miR-205 in OC cells may affect the TME in some way.

\section{Exosomes mediate the transfer of miR-205 from OC cells to macrophages and polarize macrophages to the M2 phenotype}

We previously found that circulating exosomal miR-205 was upregulated in OC patients and that its high expression was associated with OC metastasis. Studies have shown that exosomes contain a variety of bioactive molecules, including miRNAs and that the miRNA spectrum of exosomes is similar to that of the parental cells. To investigate whether exosomes mediate the transfer of miR-205 from OC cells to macrophages, we conducted a series of experiments. First, exosomes from cell-conditioned medium were isolated. As shown in Fig. 2a, the typical round particles ranged in diameter from 30 to $100 \mathrm{~nm}$ and were characterized by teacup-like shapes. Western blot analysis confirmed the presence of the exosomal proteins CD63, TSG101 and Hsp70 (Fig. 2b).

Cancer cells have been reported to secrete miRNA-carrying exosomes, and exosomes are critical for cancer initiation and progression by transferring genetic information to adjacent or distant cells.

Macrophages are among the most abundant immune-related cells recruited to the tumour site and play significant roles in tumour cell invasion and metastasis. Next, we investigated whether miR-205 derived from OC cells can be transported to macrophages through exosomes to then influence the phenotype of TAMs. The OC cell line HO-8910 with stable overexpression of miR-205 and the negative control cell line were established, and exosomes with relatively high miR-205 content (miR-205-Exos) and relatively low miR-205 content (NC-Exos) were isolated. After labelling with the fluorescent dye PKH67, miR-205-Exos from OC cells were incubated with macrophages. As shown in Fig. 2c, the PKH67-labelled exosomes could be internalized by macrophages. Next, expression levels of miR-205 in isolated exosomes were confirmed (Fig. 2d). Then, qRT-PCR was used to detect the expression of miR-205 in macrophages after treatment with NC-Exos, miR-205-Exos or PBS (as blank control). The results showed that miR-205 expression was significantly increased in macrophages treated with miR-205-Exos compared with NCExos or control (PBS) (Fig. 2e). After incubation with PMA, human THP-1 monocytes were differentiated into macrophage-like cells (Fig. 2f), during which the expression levels of the macrophage marker CD68 were significantly increased (Fig. 2g). Next, we assessed the possible role of miR-205 in OC-mediated M2 macrophage polarization via exosomes. The expression levels of M2 cytokines (CD163, IL10 and CCL8) in macrophages were significantly increased when the cells were incubated with miR-205-Exos compared with NC-Exos or control (Fig. 2h, i and j), while the expression of M1 cytokines (IL-1 $\beta$ and TNF-a) decreased significantly (Fig. $2 \mathrm{k}$ and I). Therefore, the above results demonstrate that exosomes mediate the transfer of miR-205 from OC cells to macrophages and that exosomal miR-205 from OC can induce macrophage differentiation into the M2 phenotype instead of the M1 phenotype.

Exosomal miR-205 increases the stimulatory effects of macrophages on OC cell migration, invasion and EMT 
TAMs are widely known for their crucial roles in various aspects of cancer progression, including tumour cell invasion, motility, angiogenesis and premetastatic site formation (17). Next, a co-culture system was established to investigate the effects of cancer-derived exosomal miR-205-induced M2 macrophages on the migration and invasion abilities of OC cells (Fig. 3a). As shown in Fig. 3b and C, miR-205-Exos-treated macrophages significantly increased the migration and invasion of OC cells compared to NC-Exos-treated macrophages. In addition, miR-205 mimic- or inhibitor-transfected macrophages also enhanced or weakened, respectively, the migration and invasion of OC cells (Fig. 3d and e). Next, we sought to evaluate whether exosomal miR-205-induced macrophages affect EMT in OC cells. Compared to coculture with NC-Exos-treated or NC-transfected cells, co-culture with miR-205-Exos-treated or miR-205 mimic-transfected macrophages significantly decreased epithelial cell marker (E-cadherin) expression levels and increased mesenchymal cell marker (vimentin and MMP7) expression levels, and these effects were reversed by the miR-205 inhibitor (Fig. $3 f$ and g). Taken together, these results suggest that exosomal miR-205-induced macrophages enhance the migration, invasion and EMT of OC cells.

\section{Exosomal miR-205 alters the macrophage phenotype by downregulating PTEN expression}

To deeply explore the mechanism by which exosomal miR-205 modulates macrophage polarization, TargetScan and miRBase were used to determine possible miR-205 target genes. The candidate target gene PTEN was chosen for its role in the regulation of macrophage polarization, and luciferase reporter was assays were performed in our previous report to confirm the direct targeting inhibitory effect of miR205 on PTEN. Consistent with the results of the luciferase reporter assays, miR-205 mimics or inhibitor markedly decreased or increased, respectively, the PTEN mRNA and protein levels in macrophages (Fig. $4 a$ and $b$ ). To determine the role of PTEN in M2 macrophage polarization induced by exosomal miR-205, a PTEN plasmid was used to restore PTEN expression after treatment with miR-205 mimics or miR-205Exos (Fig. 4c and d). Consistent with the previous study, overexpression of miR-205 notably upregulated the expression levels of M2-type markers (CD163 and IL10) and downregulated the levels of M1-type markers (IL-1 $\beta$ and TNF- $\alpha$ ), and these effects were reversed by PTEN expression (Fig. 4e-I).

\section{The PI3K/AKT/mTOR axis contributes to exosomal miR-205-induced M2 macrophage polarization}

PTEN is a negative regulator of the PI3K pathway and has been shown to regulate M2 macrophage polarization via the AKT/mTOR signalling pathway. Therefore, we speculated that exosomal miR-205 could promote the polarization of M2 macrophages through the PTEN-AKT-mTOR signalling pathway. To determine whether exosomal miR-205 regulates macrophage polarization through the PTEN/AKT/mTOR pathway, activation of the AKT/mTOR signalling pathway in macrophages was measured after transfection with miR-205 mimics, miR-205-Exos or PTEN plasmid. The results indicated that overexpression of miR-205 markedly increased the expression levels of p-AKT, p-mTOR and p-4EBP-1 in macrophages, and these effects were reversed by PTEN expression (Fig. 5a and b).

We then evaluated the effect of PI3K/AKT/mTOR signalling pathway-specific blockade on M2 macrophage polarization induced by exosomal miR-205. Western blot analysis confirmed that the PI3K inhibitor GDC-0941, AKT inhibitor MK-2206 and mTOR inhibitor rapamycin effectively blocked the 
activation of the PI3K/AKT/mTOR pathway induced by miR-205 upregulation (Fig. 5a and b). As shown in Fig. 5d-k, the effects of miR-205 mimics and miR-205-Exos on M2 macrophage polarization were significantly abolished by GDC-0941, MK-2206 and rapamycin. These data indicate that exosomal miR205 activates the PI3K/AKT/mTOR axis by downregulating PTEN expression in macrophages, thus inducing M2 macrophage polarization.

\section{Exosomal miR-205 promotes the development and metastasis of $\mathrm{OC}$ by inducing M2 macrophages in vivo}

Next, we further evaluated the effects of exosomal miR-205-induced M2 phenotype polarization on cancer progression. As shown in Fig. 6a, macrophages were pretreated with NC-Exos or miR-205-Exos for $48 \mathrm{~h}$. Then, luciferase-labelled HO-8910 cells mixed with the pretreated macrophages were injected into the abdominal cavities of nude mice. Subsequently, the mice were intraperitoneally injected with the same number of macrophages pretreated with NC-Exos or miR-205-Exos every three days. The IVIS system was used to detect the luciferase activity levels, which reflected the tumour volume in the peritoneal cavity. In comparison with those in the NC-Exos group, the luciferase activity levels of the miR205-Exos group increased significantly, especially at three and four weeks after the first injection (Fig. 6b and c). In addition, we found that the miR-205-Exos group produced larger tumours in the mouse peritoneal cavity than those produced in the NC-Exos group (Fig. $6 \mathrm{~d}$ ), and the number of peritoneal implants in the miR-205-Exos group was greater than that in the NC-Exos group (Fig. 6e). Compared with mice in the NC-Exos group, mice in the miR-205-Exos group showed shorter survival times and lower body weights (Fig. $6 \mathrm{f}$ and g). IHC and HE staining showed that the expression levels of CD163 (an M2-type macrophage marker) were significantly increased in the miR-205-Exos group compared with the NC-Exos group (Fig. $6 \mathrm{~h}$ and i), suggesting that there were more M2 macrophages infiltrating around the cancer tissues in the miR-205-Exos group than in the NC-Exos group. These results indicate that exosomal miR205 induces M2-type polarization of macrophages in the TME and promotes OC progression and metastasis in vivo.

\section{Discussion}

With the increased popularity of clinical immunotherapy in recent years, the regulation of the immune microenvironment in $\mathrm{OC}$ has gained increasing attention [28-30]. TAMs, an important part of the TME, have also become a primary drug target for tumour therapy [31, 32]. Many studies report that the communication between $\mathrm{OC}$ cells and macrophages could be mediated by exosomes, and exosomal miRNAs released from cancer cells could definitely reprogram macrophages to establish a kind of tumour immunosuppressive microenvironment that may promote the invasion and metastasis of tumour cells [33-37]. Our previous studies showed that upregulation of miR-205 contributes to OC progression and that circulating exosomal miR-205 is associated with OC metastasis [24]. In this study, we explored the role and underlying mechanisms of exosomal miR-205 in the progression of OC. We found that miR-205 expression was associated with TAM infiltration, and OC-derived miR-205 could induce M2 macrophage polarization via exosomes. Moreover, M2 macrophages induced by exosomal miR-205 promoted cancer 
progression via the PI3K/AKT/mTOR axis (Fig. 7). In conclusion, these data indicate the important role of OC-derived exosomal miR-205 in the antitumour immunologic reaction of OC and its potential as a mediator of cancer progression.

TAMs are the main immune cells in the TME and play a significant role in cancer development and metastasis [38, 39]. TAMs are mainly divided into antitumour macrophages (M1-type macrophages) and tumour-promoting macrophages (M2-type macrophages). M1-type and M2-type macrophages can transform each other under certain conditions [40]. In the early stage of tumour formation, TAMs are dominated by the M1 type, and with tumour development they gradually transformed into the M2 type. M1-type macrophages secrete pro-inflammatory cytokines and chemokines to participate in antigen presentation and antitumour immune responses, while M2-type macrophages are believed to be the main phenotype of TAMs and secrete inhibitory cytokines with tumour-promoting functions [41]. M2-type macrophages, which have lost their cytotoxic capacity, participate in a variety of cancer-promoting activities, including cancer-related inflammation, immunosuppression, and metastasis [42- 44]. Here, we found that the expression level of miR-205 was upregulated in patients with OC and positively associated with M2-type macrophage infiltration. Furthermore, it is interesting to note that miR-205 staining was observed in both tumour cells and CD68+ and CD163+ cells in most of the OC specimens, suggesting that miR-205 may participate in the regulation of TAMs.

Exosomes are important components of the TME and act as an important messenger that mediates the cross-talk between tumour cells and immune cells [45]. The communication between tumour cells and immune cells via exosomes is considered to be an effective mechanism that plays a significant role in the establishment of the tumour immune microenvironment (TIME), thereby promoting cancer progression [46]. A large number of studies have suggested that exosomal miRNAs could dramatically influence the phenotypic transformation of macrophages through various targets and that exosomal miRNA release from cancer cells could definitely promote the invasion and metastasis of tumour cells [47-49]. miR-205 has been reported to function as a cancer-promoting miRNA in many cancers, and its overexpression in circulating exosomes was associated with OC metastasis [23, 24]. In this study, we demonstrated that miR-205 was significantly enriched in exosomes derived from OC cells and could be transferred from OC cells to macrophages via exosomes. Therefore, OC cell-derived exosomal miR-205 could induce the alternative activation of macrophages towards the M2 phenotype, which promotes the migration, invasion, and EMT of OC cells. These results suggest that OC cell-derived exosomal miR-205 plays an important role in the polarization of macrophages in the TME and is important for tumour cell invasion and metastasis.

From a list of predicted target genes of miR-205, PTEN was chosen for its function in the $\mathrm{PIBK} / \mathrm{AKT} / \mathrm{mTOR}$ pathway. PTEN is a key mediator of the PI3K/AKT/mTOR pathway, which plays an essential role in the activation and polarization of macrophages [20,50,51]. However, the regulatory mechanism of this link has not been clear so far, so targeted therapy cannot be developed. Therefore, it is of great clinical significance to further explore the molecular mechanism of the regulation of TAM phenotypic polarization for the immune treatment of OC. In this study, our results indicated that OC- 
derived exosomal miR-205 inhibited the expression of PTEN in macrophages and then activated the $\mathrm{PI} 3 \mathrm{~K} / \mathrm{AKT} / \mathrm{mTOR}$ axis. By binding to the $3^{\prime}$ UTR of target genes, miRNAs function as negative regulators of gene expression and degrade the expression of target mRNAs. Our previous study demonstrated that miR-205 can decrease the expression level of PTEN by directly targeting the 3' UTR of PTEN mRNA [24]. Our data also showed that exosomal miR-205 inhibited the expression level of the PTEN protein and mRNA. Next, the decreased expression of PTEN, modulated by exosomal miR-205, increased the expression of p-AKT, p-mTOR, p-4EBP-1 and p-p70-S6 in macrophages, which resulted in activation of the $\mathrm{PI} 3 \mathrm{~K} / \mathrm{AKT} / \mathrm{mTOR}$ signalling pathway. In contrast, restoration of PTEN expression inhibited the activation of the PI3K/AKT/mTOR pathway and the expression of immunosuppressive factors induced by exosomal miR-205, revealing that miR-205 promotes the polarization of M2 macrophages by silencing PTEN and subsequently activating its downstream PI3K/AKT/mTOR pathway.

According to recent studies, the PI3K/AKT/mTOR axis is involved in the regulation of cancer progression by promoting the polarization of macrophages and mediating immune suppression [52]. By regulating transcriptional programming of M2 macrophages, mTOR signalling suppresses immune responses and promotes cancer development and metastasis [53]. To further explore the role of the PI3K/AKT/mTOR pathway in the polarization of macrophages mediated by exosomal miR-205, inhibitors of the $\mathrm{PI} 3 \mathrm{~K} / \mathrm{AKT} / \mathrm{mTOR}$ pathway were used. Here, the PI3K, AKT and mTOR inhibitors significantly blocked the phenotypic switch of macrophages towards the M2 type induced by exosomal miR-205. In addition, the activation of the PI3K/AKT/mTOR axis by exosomal miR-205 promoted the differentiation and polarization of M2 macrophages, leading to tumour growth, metastasis and poor prognosis in assays in vivo.

\section{Conclusions}

In conclusion, we demonstrated that miR-205 expression was associated with TAM infiltration in OC, and OC cell-derived exosomal miR-205 induced macrophage M2 polarization by regulating the PTEN/PI3K/AKT/mTOR axis, which promoted cancer development and metastasis in vivo and in vitro. These findings reveal the underlying molecular mechanisms of $\mathrm{OC}$ immunoregulation in the TIME and may contribute to the development of better clinical immune treatment for patients with OC.

\section{Abbreviations}

CD206: macrophage mannose receptors 206; CD163: macrophage mannose receptors 163; EMT: epithelial-mesenchymal transition; FBS: foetal bovine serum; ISH: in situ hybridization; IHC: immunohistochemistry; miRNAs: microRNAs; IL-6: interleukin-6; IL-1ß: interleukin-1 $\beta$; IL10: interleukin-10; IL8: interleukin-8; miRNAs: microRNAs; OC: ovarian cancer; PBS: phosphate-buffered saline; PMA: phorbol 12-myristate 13-acetate; TEM: transmission electron microscopy; TAMs: Tumour-associated macrophages; TME: tumour microenvironment; IFN- $\gamma$ : interferon- $\gamma ;$ TNF-a: tumor necrosis factor-a.

\section{Declarations}




\section{Ethics approval and consent to participate}

The present study was approved by the Ethics Committee of Xiangya Hospital ( Central South University, Changsha, China) and conducted in adherence with the Declaration of Helsinki. The patients were informed about the sample collection and had signed informed consent forms.

\section{Consent for publication}

All authors of this article have directly participated in the planning and drafting and all authors listed have read and approved the final version including details and images. The written informed consent for the publication has been obtained from all the authors. The patients were informed about the publication and had signed informed consent forms.

\section{Availability of data and materials}

The analyzed and/or datasets generated during the study are available from the corresponding author on reasonable request.

\section{Competing interests}

The authors declare that they have no competing interests.

\section{Funding}

This work was supported by grants from the National Natural Science Foundation of China (grant no. 81172469), the Open Sharing Fund for the Large-scale Instruments and Equipments of Central South University (grant no. CSUZC202039) and Hunan Provincial Natural Science Foundation of China (grant no. 2019JJ40394).

\section{Authors' contributions}

LQH conceived, designed the study, prepared all the figures and wrote the manuscript. QC and DW contributed to the study design and performed the experiments. WZ and QFC provided technical support and analysed the data. XYW contributed to the study design and reviewed and edited the manuscript. All authors read and approved the final manuscript.

\section{Acknowledgements}

Not applicable.

\section{References}

1. Torre LA, Trabert B, DeSantis CE, Miller KD, Samimi G, Runowicz CD, Gaudet MM, Jemal A, Siegel RL. Ovarian cancer statistics, 2018. CA Cancer J Clin. 2018;68(4):284-96.

2. Siegel RL, Miller KD, Jemal A. Cancer statistics, 2020. CA Cancer J Clin. 2020;70(1):7-30.

3. Beltraminelli T. Biology and therapeutic targeting of tumour-associated macrophages. J Pathol. 2020;250(5):573-92., De Palma M.

4. Tian $\mathrm{K}$, Wang $\mathrm{X}$, Wu Y, Wu X, Du G, Liu W, Wu R. Relationship of tumour-associated macrophages with poor prognosis in Wilms' tumour. J Pediatr Urol. 2020;16(3):376 e1-8. 
5. Wu H, Zhang X, Han D, Cao J, Tian J. Tumour-associated macrophages mediate the invasion and metastasis of bladder cancer cells through CXCL8. PeerJ. 2020;12(8):e8721.

6. Zhu Z, Zhu X, Yang S, Guo Z, Li K, Ren C, Zhou Y, Dou J. Yin-yang effect of tumour cells in breast cancer: from mechanism of crosstalk between tumour-associated macrophages and cancerassociated adipocytes. Am J Cancer Res. 2020;10(2):383-92.

7. De Palma M, Lewis CE: Macrophage regulation of tumor responses to anticancer therapies. Cancer Cell. 2013; 23(3):277-86.

8. Martinez FO, Gordon S. The M1 and M2 paradigm of macrophage activation: time for reassessment. F1000Prime Rep. 2014;6(13):6-13.

9. Murray PJ, Allen JE, Biswas SK, Fisher EA, Gilroy DW, Goerdt S, Gordon S, Hamilton JA, Ivashkiv LB, Lawrence T. et al: Macrophage activation and polarization: nomenclature and experimental guidelines. Immunity. 2014;41(1):14-20.

10. Curtale G. MiRNAs at the Crossroads between Innate Immunity and Cancer: Focus on Macrophages. Cells. 2018;7(2):12.

11. Su S, Liu Q, Chen J, Chen J, Chen F, He C, Huang D, Wu W, Lin L, Huang W. et al: A positive feedback loop between mesenchymal-like cancer cells and macrophages is essential to breast cancer metastasis. Cancer Cell. 2014;25(5):605-20.

12. Diaferia GR, Natoli G. When antimicrobial peptides hit the wrong target: a novel link between tumour macrophages and cancer stem cells. Gut. 2015;64(12):1841-2.

13. Conway EM, Pikor LA, Kung SH, Hamilton MJ, Lam S, Lam WL, Bennewith KL. Macrophages, Inflammation, and Lung Cancer. Am J Respir Crit Care Med. 2016;193(2):116-30.

14. Xu Z, Zeng S, Gong Z, Yan Y. Exosome-based immunotherapy: a promising approach for cancer treatment. Mol Cancer. 2020;19(1):160.

15. Zang X, Gu J, Zhang J, Shi H, Hou S, Xu X, Chen Y, Zhang Y, Mao F, Qian H. et al: Exosometransmitted IncRNA UFC1 promotes non-small-cell lung cancer progression by EZH2-mediated epigenetic silencing of PTEN expression. Cell Death Dis. 2020;11:215.

16. Zhao L, Gu C, Gan Y, Shao L, Chen H, Zhu H. Exosome-mediated siRNA delivery to suppress postoperative breast cancer metastasis. J Control Release. 2020;318(12):1-15.

17. Wang D, Wang X, Si M, Yang J, Sun S, Wu H, Cui S, Qu X, Yu X. Exosome-encapsulated miRNAs contribute to CXCL12/CXCR4-induced liver metastasis of colorectal cancer by enhancing M2 polarization of macrophages. Cancer Lett. 2020;474(4):36-52.

18. Yu S, Sha H, Qin X, Chen Y, Li X, Shi M, Feng J. EGFR E746-A750 deletion in lung cancer represses antitumor immunity through the exosome-mediated inhibition of dendritic cells. Oncogene. 2020;39(13):2643-57.

19. Jiang M, Zhang W, Zhang R, Liu P, Ye Y, Yu W, Guo X, Yu J. Cancer exosome-derived miR-9 and miR181a promote the development of early-stage MDSCs via interfering with SOCS3 and PIAS3 respectively in breast cancer. Oncogene. 2020;39(24):4681-94. 
20. Xiao L, He Y, Peng F, Yang J, Yuan C. Endometrial Cancer Cells Promote M2-Like Macrophage Polarization by Delivering Exosomal miRNA-21 under Hypoxia Condition. J Immunol Res. 2020; 2020(10): 9731049.

21. Zhao S, Mi Y, Guan B, Zheng B, Wei P, Gu Y, Zhang Z, Cai S, Xu Y, Li X. et al: Tumor-derived exosomal miR-934 induces macrophage M2 polarization to promote liver metastasis of colorectal cancer. $J$ Hematol Oncol. 2020;13(1):156.

22. Tong F, Mao X, Zhang S, Xie H, Yan B, Wang B, Sun J, Wei L. HPV + HNSCC-derived exosomal miR-9 induces macrophage $\mathrm{M} 1$ polarization and increases tumor radiosensitivity. Cancer Lett. 2020;478(5):34-44.

23. Li J, Hu K, Gong G, Zhu D, Wang Y, Liu H, Wu X. Upregulation of MiR-205 transcriptionally suppresses SMAD4 and PTEN and contributes to human ovarian cancer progression. Sci Rep. 2017;7(12):41330.

24. He L, Zhu W, Chen Q, Yuan Y, Wang Y, Wang J, Wu X. Ovarian cancer cell-secreted exosomal miR-205 promotes metastasis by inducing angiogenesis. Theranostics. 2019;9(26):8206-20.

25. He L, Zhu D, Wang J, Wu X. A highly efficient method for isolating urinary exosomes. Int J Mol Med. 2019;43(1):83-90.

26. Zhou J, Gong G, Tan H, Dai F, Zhu X, Chen Y, Wang J, Liu Y, Chen P, Wu X, Wen J. Urinary microRNA$30 a-5 p$ is a potential biomarker for ovarian serous adenocarcinoma. Oncol Rep. 2015;33(6):291523.

27. Li J, Li L, Li Z, Gong G, Chen P, Liu H, Wang J, Liu Y, Wu X. The role of miR-205 in the VEGF-mediated promotion of human ovarian cancer cell invasion. Gynecol Oncol. 2015;137(1):125-33.

28. Gao Y, Chen L, Cai G, Xiong X, Wu Y, Ma D, Li SC, Gao Q. Heterogeneity of immune microenvironment in ovarian cancer and its clinical significance: a retrospective study. Oncoimmunology. 2020;9(1):1760067.

29. Fan S, Gao X, Qin Q, Li H, Yuan Z, Zhao S. Association between tumor mutation burden and immune infiltration in ovarian cancer. Int Immunopharmacol. 2020;89:107126. (Pt A).

30. Ghisoni E, Imbimbo M, Zimmermann S, Valabrega G. Ovarian Cancer Immunotherapy: Turning up the Heat. Int J Mol Sci. 2019;20(12):2927-43.

31. Cassetta L, Pollard JW. Targeting macrophages: therapeutic approaches in cancer. Nat Rev Drug Discov. 2018;17(12):887-904.

32. De Beule N, De Veirman K, Maes K. De Bruyne E, Menu E, Breckpot K, De Raeve H, Van Rampelbergh R, Van Ginderachter JA, Schots R, et al: Tumour-associated macrophage-mediated survival of myeloma cells through STAT3 activation. J Pathol. 2017; 241(4):534-46.

33. Chen C, Liu J-m. Luo Y-p: MicroRNAs in tumor immunity: functional regulation in tumor-associated macrophages. Journal of Zhejiang University-SCIENCE B. 2019;21(1):12-28.

34. Lan J, Sun L, Xu F, Liu L, Hu F, Song D, Hou Z, Wu W, Luo X, Wang J. et al: M2 Macrophage-Derived Exosomes Promote Cell Migration and Invasion in Colon Cancer. Cancer Res. 2019;79(1):146-58. 
35. Li N, Qin J-F, Han X, Jin F-J, Zhang J-H, Lan L, Wang Y. miR-21a negatively modulates tumor suppressor genes PTEN and miR-200c and further promotes the transformation of M2 macrophages. Immunol Cell Biol. 2018;96(1):68-80.

36. Qian M, Wang S, Guo X, Wang J, Zhang Z, Qiu W, Gao X, Chen Z, Xu J, Zhao R. et al: Hypoxic gliomaderived exosomes deliver microRNA-1246 to induce M2 macrophage polarization by targeting TERF2IP via the STAT3 and NF-KB pathways. Oncogene. 2019;39(2):428-42.

37. Hsu YL, Hung JY, Chang WA, Jian SF, Lin YS, Pan YC, Wu CY, Kuo PL. Hypoxic Lung-Cancer-Derived Extracellular Vesicle MicroRNA-103a Increases the Oncogenic Effects of Macrophages by Targeting PTEN. Mol Ther. 2018;26(2):568-81.

38. Wei C, Yang C, Wang S, Shi D, Zhang C, Lin X, Liu Q, Dou R, Xiong B. Crosstalk between cancer cells and tumor associated macrophages is required for mesenchymal circulating tumor cell-mediated colorectal cancer metastasis. Mol Cancer. 2019;18(1):64.

39. Guo Q, Jin Z, Yuan Y, Liu R, Xu T, Wei H, Xu X, He S, Chen S, Shi Z, et al: New Mechanisms of TumorAssociated Macrophages on Promoting Tumor Progression: Recent Research Advances and Potential Targets for Tumor Immunotherapy. J Immunol Res. 2016; 2016:9720912.

40. Ngambenjawong C, Gustafson HH, Pun SH. Progress in tumor-associated macrophage (TAM)targeted therapeutics. Adv Drug Deliv Rev. 2017;114(5):206-21.

41. Linde N, Casanova-Acebes M, Sosa MS, Mortha A, Rahman A, Farias E, Harper K, Tardio E, Reyes Torres I, Jones J. et al: Macrophages orchestrate breast cancer early dissemination and metastasis. Nat Commun. 2018;9(1):21.

42. Chen C, He W, Huang J, Wang B, Li H, Cai Q, Su F, Bi J, Liu H, Zhang B. et al: LNMAT1 promotes lymphatic metastasis of bladder cancer via CCL2 dependent macrophage recruitment. Nat Commun. 2018;9(1):3826.

43. Chen XW, Yu TJ, Zhang J, Li Y, Chen HL, Yang GF, Yu W, Liu YZ, Liu XX, Duan CF. et al: CYP4A in tumor-associated macrophages promotes pre-metastatic niche formation and metastasis. Oncogene. 2017;36(35):5045-57.

44. Qian BZ, Pollard JW. Macrophage diversity enhances tumor progression and metastasis. Cell. 2010;141(1):39-51.

45. Feng W, Dean DC, Hornicek FJ, Shi H, Duan Z. Exosomes promote pre-metastatic niche formation in ovarian cancer. Mol Cancer. 2019;18(1):124.

46. Xie F, Xu M, Lu J, Mao L, Wang S. The role of exosomal PD-L1 in tumor progression and immunotherapy. Mol Cancer. 2019;18(1):146.

47. Cooks T, Pateras IS, Jenkins LM, Patel KM, Robles Al, Morris J, Forshew T, Appella E, Gorgoulis VG, Harris CC. Mutant p53 cancers reprogram macrophages to tumor supporting macrophages via exosomal miR-1246. Nat Commun. 2018;9(1):771.

48. Hsieh $\mathrm{CH}$, Tai SK, Yang MH. Snail-overexpressing Cancer Cells Promote M2-Like Polarization of Tumor-Associated Macrophages by Delivering MiR-21-Abundant Exosomes. Neoplasia. 2018;20(8):775-88. 
49. Kanlikilicer P, Bayraktar R, Denizli M, Rashed MH, Ivan C, Aslan B, Mitra R, Karagoz K, Bayraktar E, Zhang $X$. et al: Exosomal miRNA confers chemo resistance via targeting Cav1/p-gp/M2-type macrophage axis in ovarian cancer. EBioMedicine. 2018;38(12):100-12.

50. Kaneda MM, Messer KS, Ralainirina N, Li H, Leem CJ, Gorjestani S, Woo G, Nguyen AV, Figueiredo CC, Foubert P. et al: PI3Kgamma is a molecular switch that controls immune suppression. Nature. 2016;539(7629):437-42.

51. Kaneda MM, Cappello P, Nguyen AV, Ralainirina N, Hardamon CR, Foubert P. et al. Macrophage PI3Kgamma drives pancreatic ductal adenocarcinoma progression. Cancer Discov. 2016;6(8):870955.

52. Pfeifer M, Grau M, Lenze D, Wenzel SS, Wolf A, Wollert-Wulf B, Dietze K, Nogai H, Storek B, Madle H. et al: PTEN loss defines a PI3K/AKT pathway-dependent germinal center subtype of diffuse large Bcell lymphoma. Proc Natl Acad Sci U S A. 2013;110(30):12420-5.

53. Rocher C, Singla DK. SMAD-PI3K-Akt-mTOR pathway mediates BMP-7 polarization of monocytes into M2 macrophages. PLoS One. 2013;8(12):e84009.

\section{Figures}



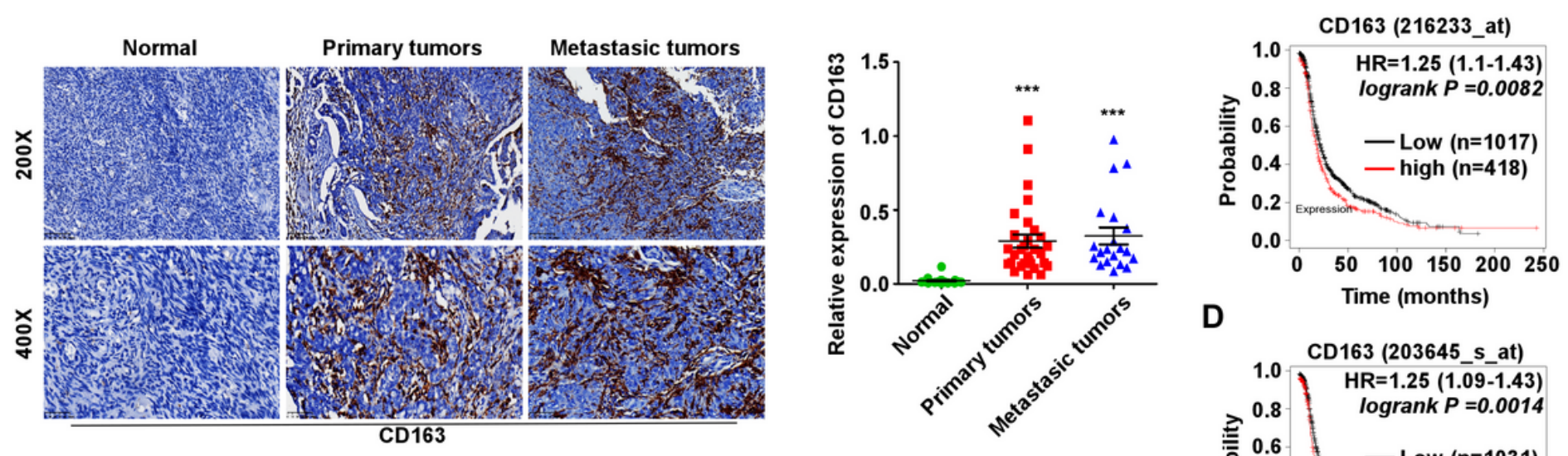

E

$\mathbf{F}$
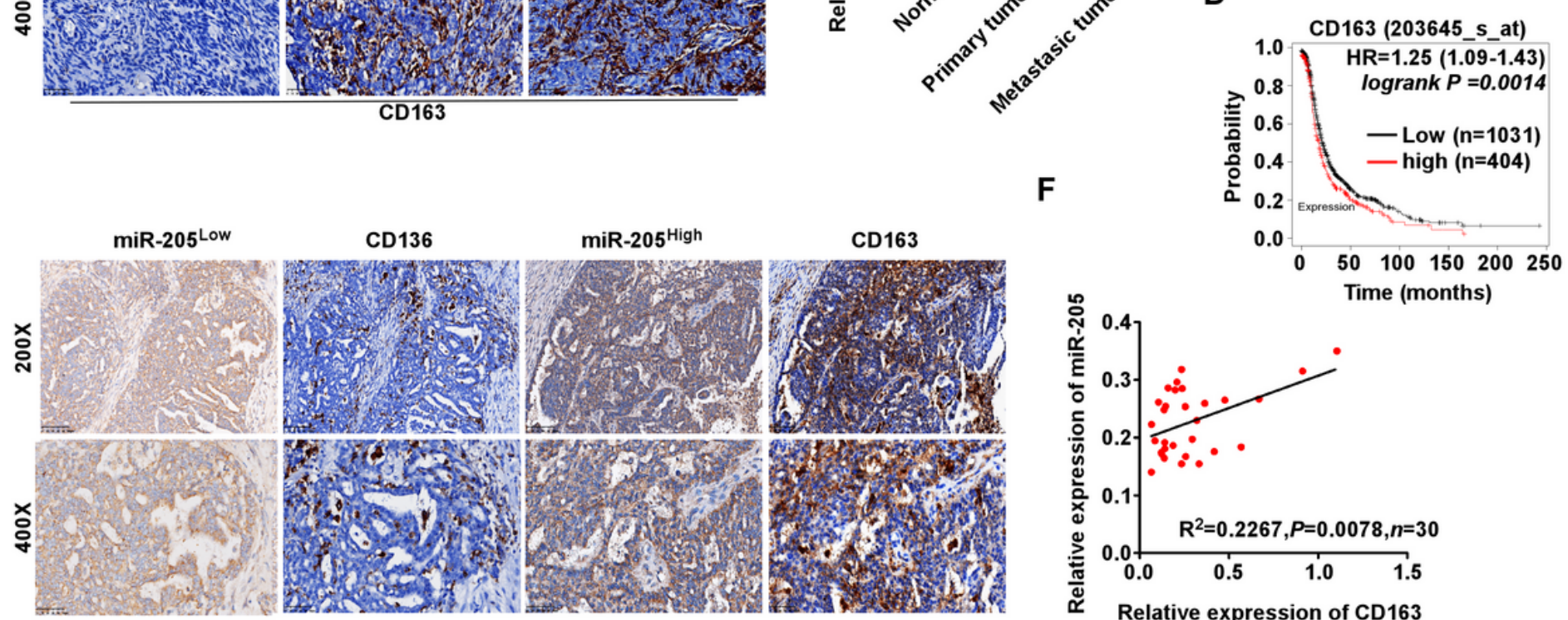

miR-205 ${ }^{\text {High }}$

CD163

G

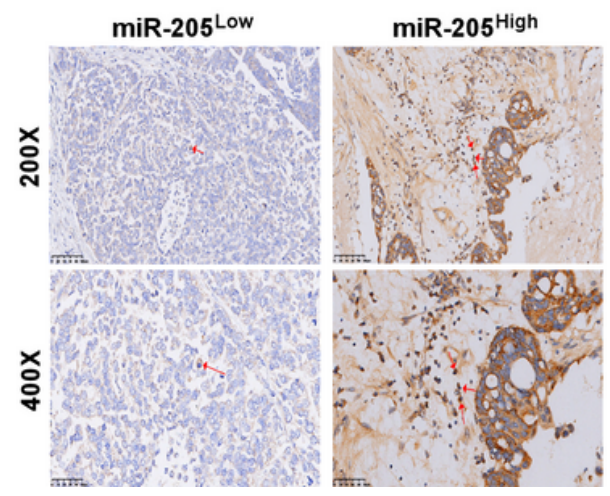

H

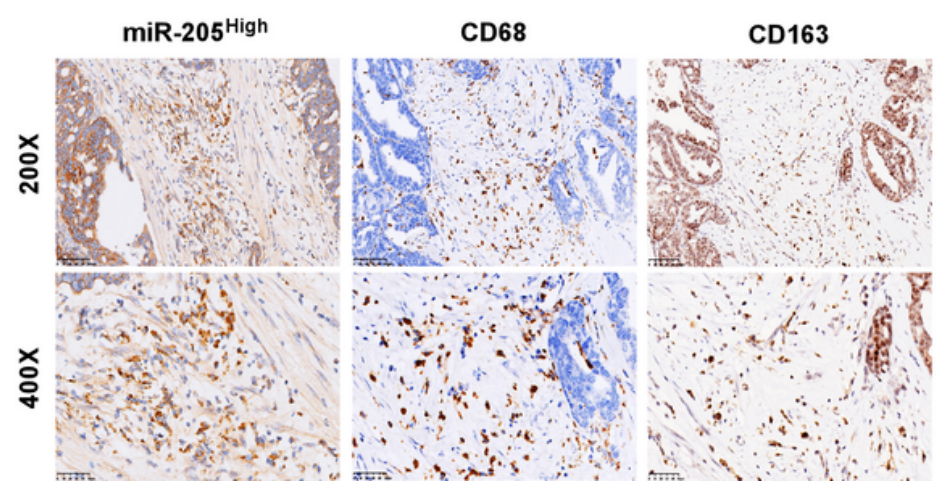

\section{Figure 1}

miR-205 expression is associated with TAM infiltration in patients with OC. a IHC was used to detect the expression of the M2 marker CD163 in normal ovarian tissues, primary tumours and specimens (black arrows). Scale bar, $100 \mathrm{~nm}$. b The relative expression of CD163 was determined in each group. c, d Kaplan-Meier curves for overall survival in OC using the Kaplan-Meier Plotter database (www.kmplot.com). Red and black lines indicate patients with higher and lower than the median CD163 expression, respectively. Data are presented as the mean $\pm S D$ and were analysed with Student's $t$ test. *** $\mathrm{P}<<0.001$. e Representative images of $\mathrm{IHC}$ for CD163 with high or low expression levels of miR-205. $f$ Spearman correlation between miR-205 expression and CD163 in OC tissues. The Pearson correlation coefficient ( $r$ ) and P-value are shown, $n=30$. g Representative images of miR-205 measured by ISH in OC 
tissues. Red arrow indicates macrophages. h Representative IHC images of CD68 and CD163 in the context of relatively high expression levels of miR-205. The scale bar in the 200x images represents 100 $\mu \mathrm{m}$. The scale bar in the $400 \times$ images represents $50 \mu \mathrm{m}$.

A

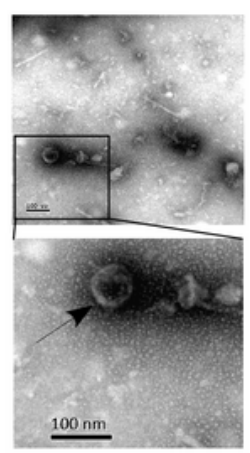

B

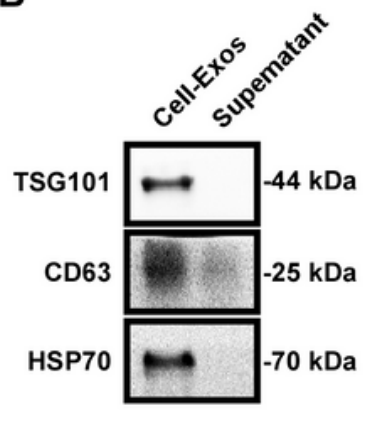

C

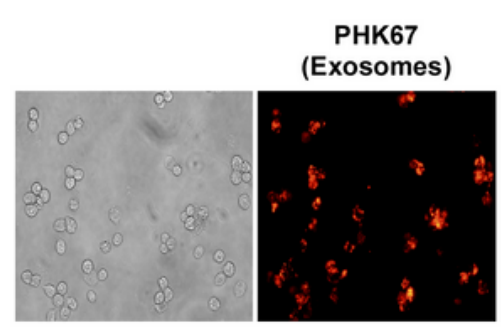

E

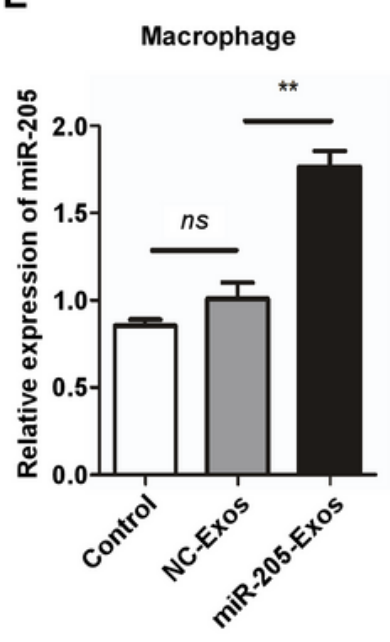

I

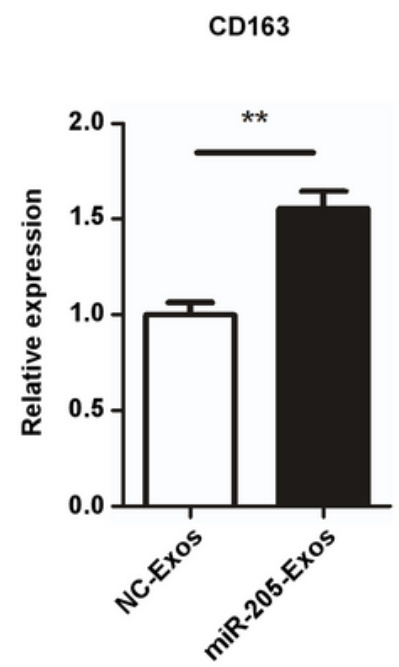

$\mathbf{F}$

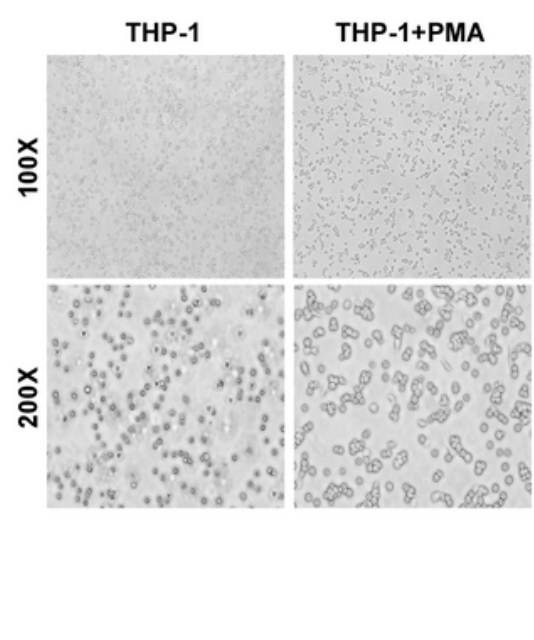

$\mathbf{J}$
G

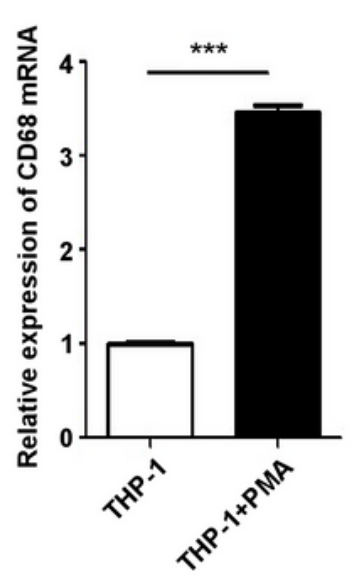

$\mathbf{K}$
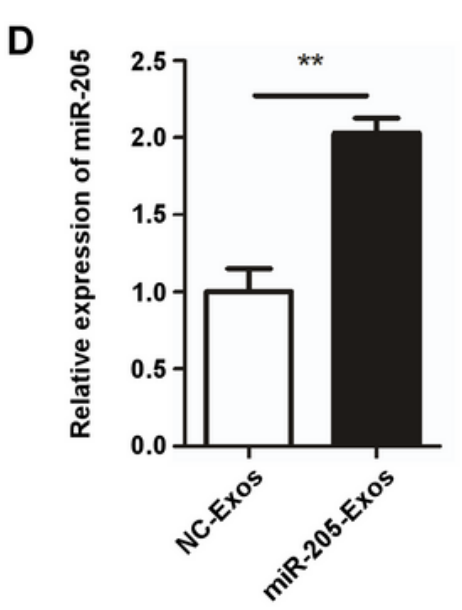

H

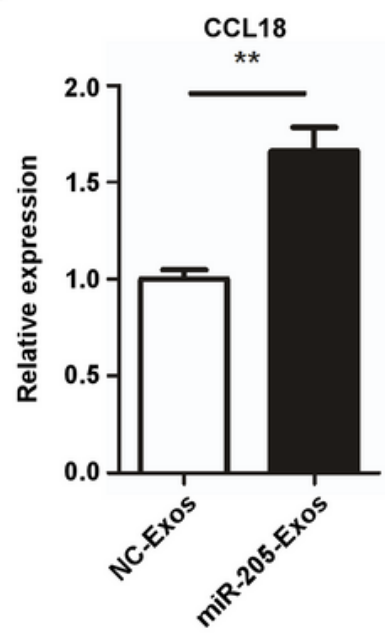

$\mathbf{L}$

IL-1 $\beta$

TNF- $\alpha$
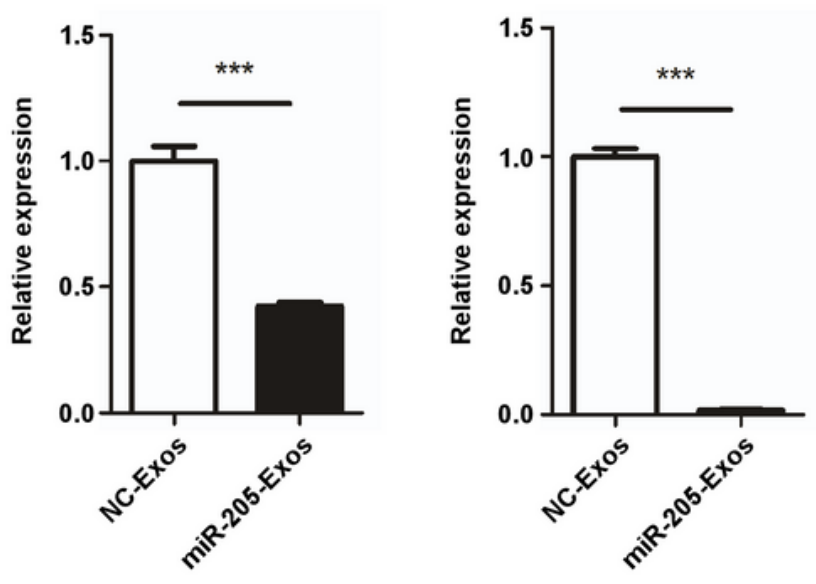

Figure 2

miR-205 can be transported from OC cells to macrophages via exosomes to alter the macrophage phenotype. a Isolated exosomes were identified under electron microscopy (black arrows). Scale bar, 100 
nm. b The exosome-specific marker TSG101 and extracellular vesicle-related protein markers Hsp70 and CD63 were measured by Western blotting analysis. Cell exosomes: exosomes isolated from cell culture medium; supernatant: supernatant after the last centrifugation. c Exosomes from HO-8910-miR-205 cells were labelled with PKH26 and then added to the macrophage culture medium. $d$ The miR-205 levels in miR-205-Exos and NC-Exos were measured by RT-PCR. e After treatment with miR-205-Exos, NC-Exos and control (as blank control), the miR-205 levels in macrophage were measured by RT-PCR. $f$ THP- 1 cells were treated with phorbol-12-myristate-13-acetate (PMA) for $24 \mathrm{~h}$. g After treatment with PMA, the expression levels of CD68 in THP-1 cells were detected by RT-PCR. h-j Effects of miR-205-Exos and NCExos on the expression levels of M2-type markers (CD163, IL10 and CCL8) were analysed by qRT-PCR in macrophages. k, I M1-type marker (IL-1 $\beta$ and TNF-a) levels were detected by RT-PCR after treatment with miR-205-Exos and NC-Exos. All data are shown as the mean \pm SEM from at least three independent experiments. ${ }^{*} \mathrm{P}<0.05,{ }^{*} \mathrm{P}<0.01$ and ${ }^{\star * *} \mathrm{P}<0.001$, Student's $t$ test. n.s, not significant. 
A

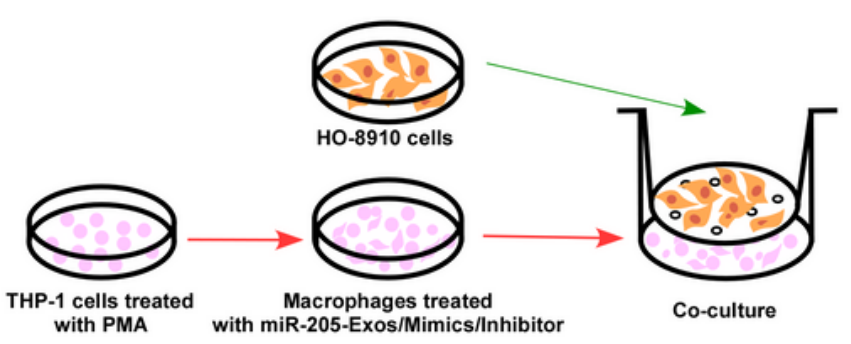

B

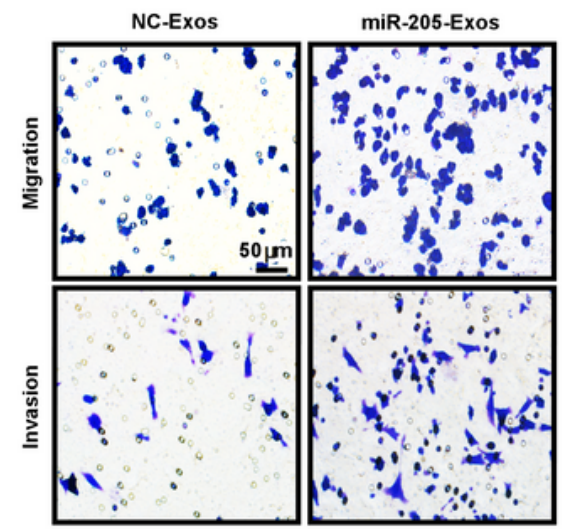

D

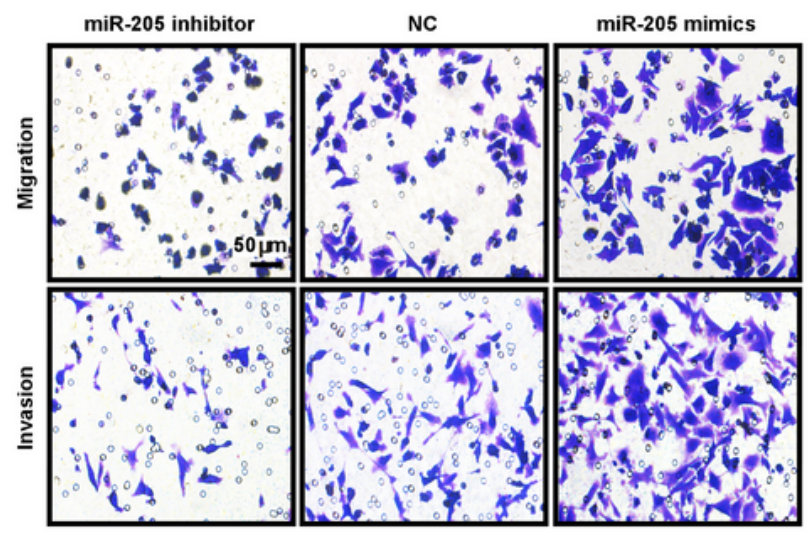

C
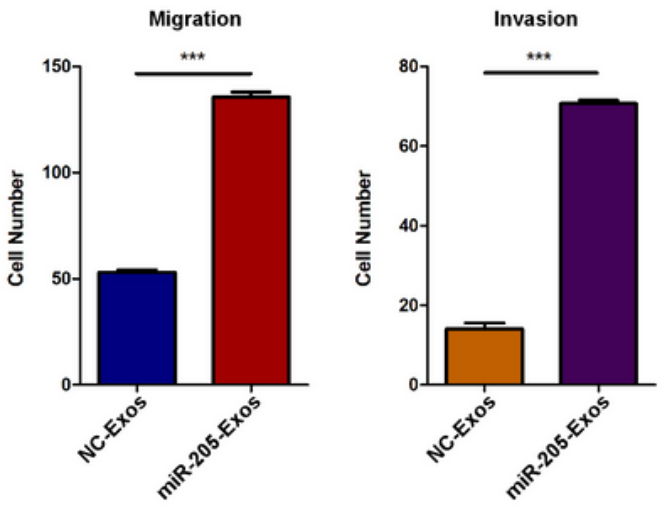

E
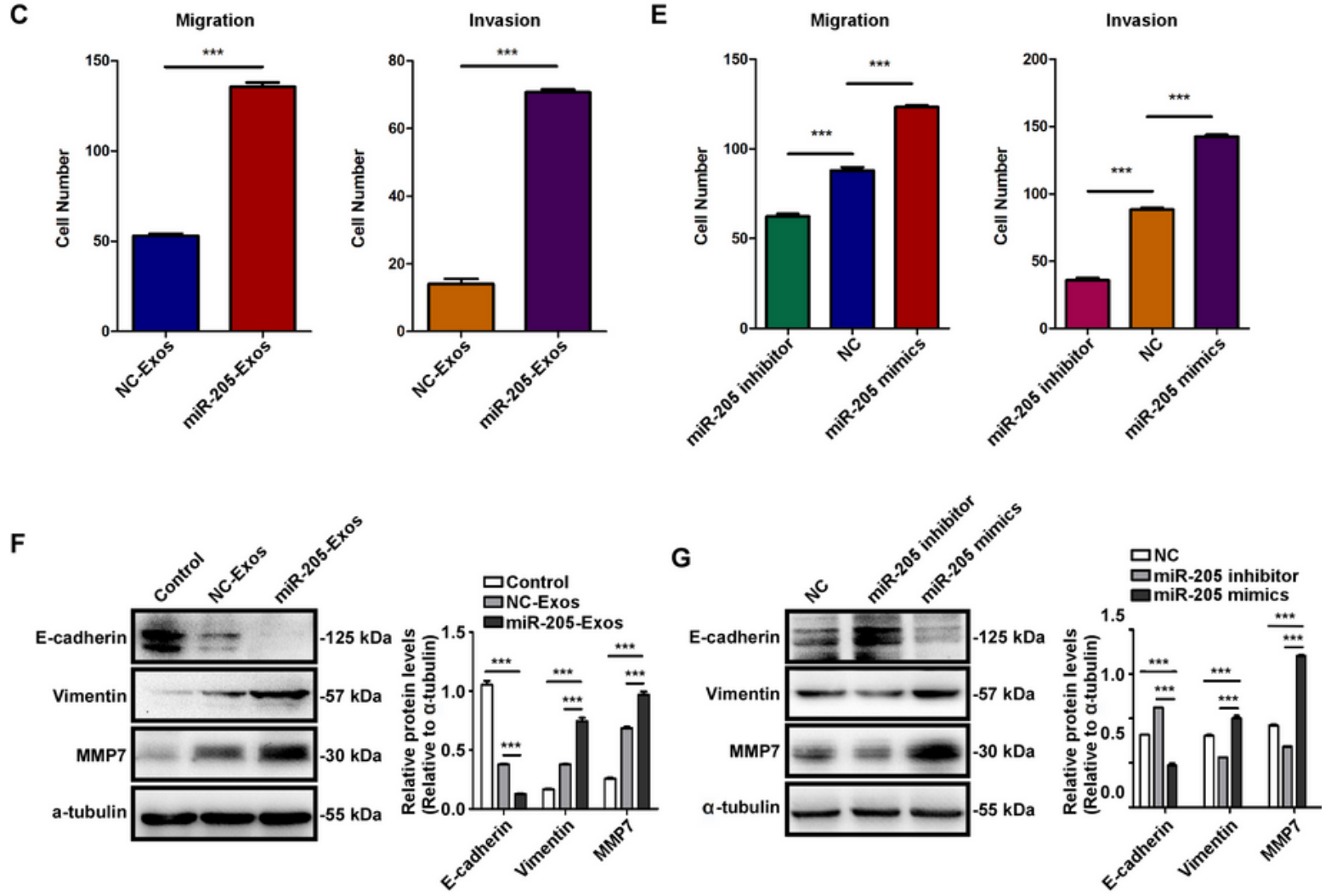

Figure 3

Exosomal miR-205-induced macrophages increased OC cell migration, invasion, and EMT. a Schematic illustration of the in vitro indirect co-culture system. $b, d$ The migration and invasion capacity of OC cells (HO-8910) co-cultured with macrophages treated with exosomes (miR-205-Exos, NC-Exos and control (as blank control)) or rmiR-205 mimics/NC/inhibitor was determined by the in vitro Transwell co-culture system. Representative images of migratory or invaded cells on membranes coated with or without 
Matrigel are shown. The scale bar represents $50 \mu \mathrm{m}$. c, e Morphometric analysis of migratory and invaded cells. $f, g$ The effect of the supernatants of macrophages treated with exosomes or rmiR-205 mimics/NC/inhibitor on the EMT of HO-8910 cells was analysed by Western blotting. All data are shown as the mean \pm SEM from at least three independent experiments. ${ }^{* * *} \mathrm{P}<0.001$, Student's $t$ test.
A
B
C
D
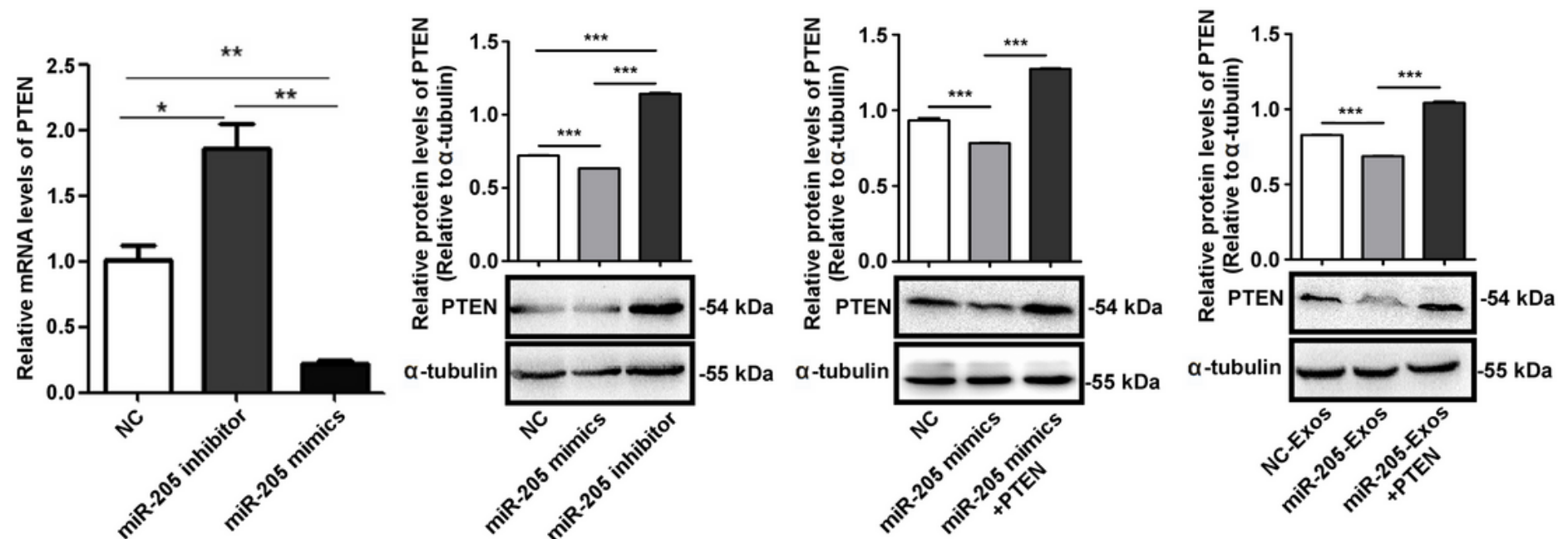

E

$\mathbf{F}$
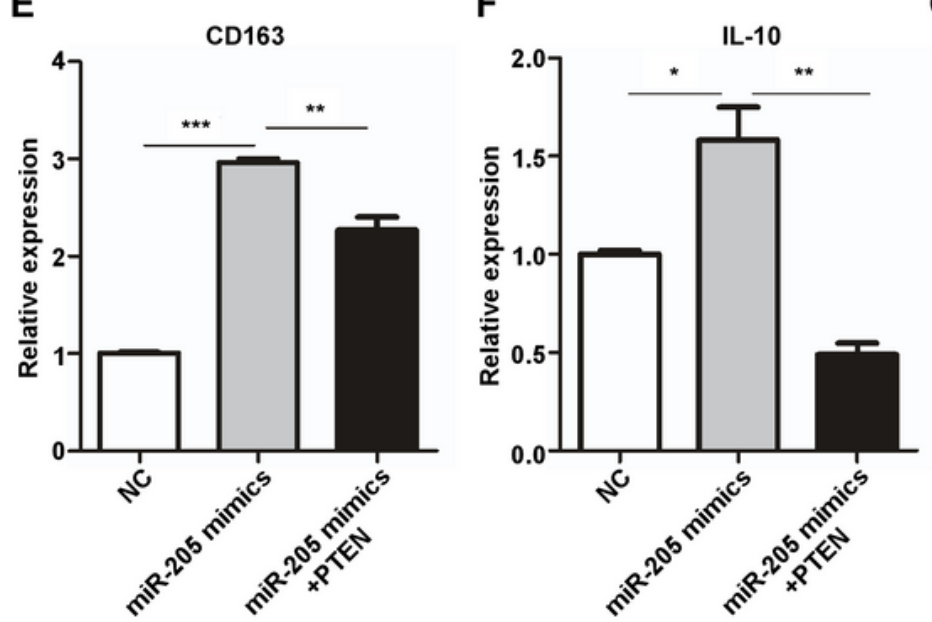

G

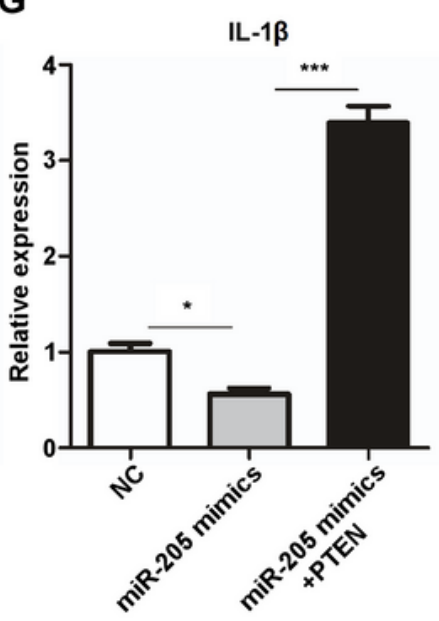

H

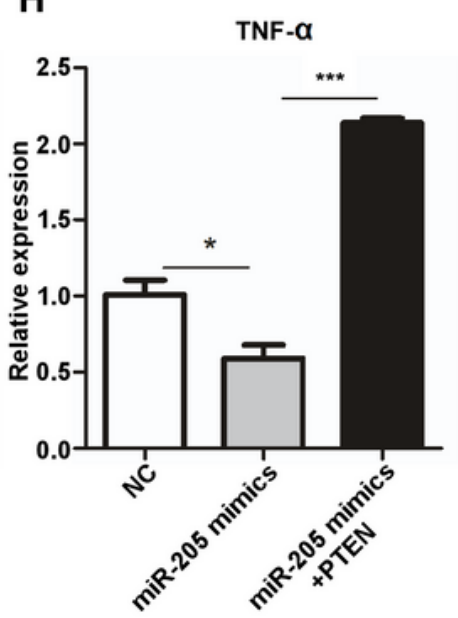

I

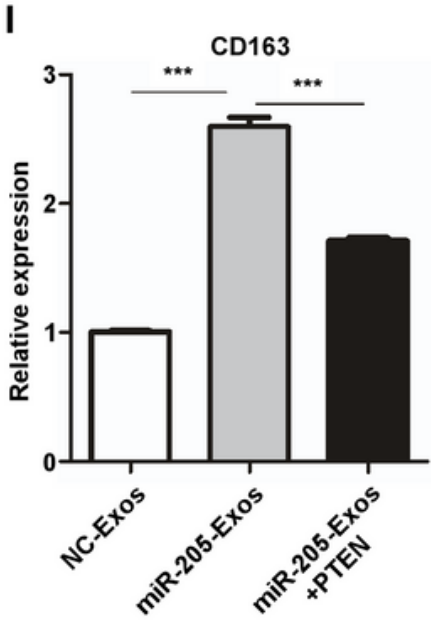

J

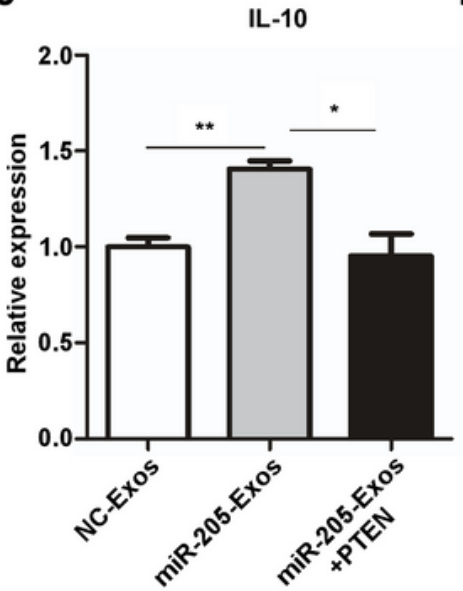

K

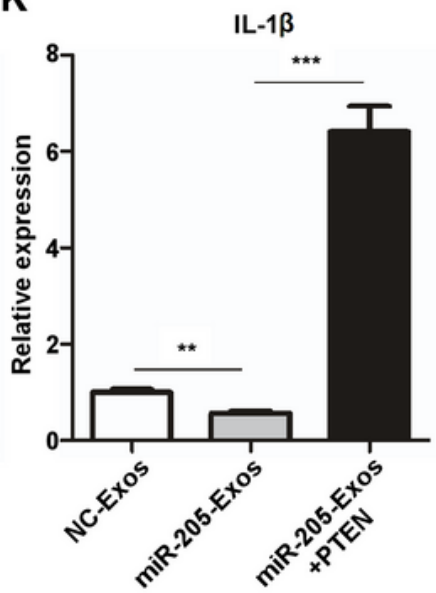

L

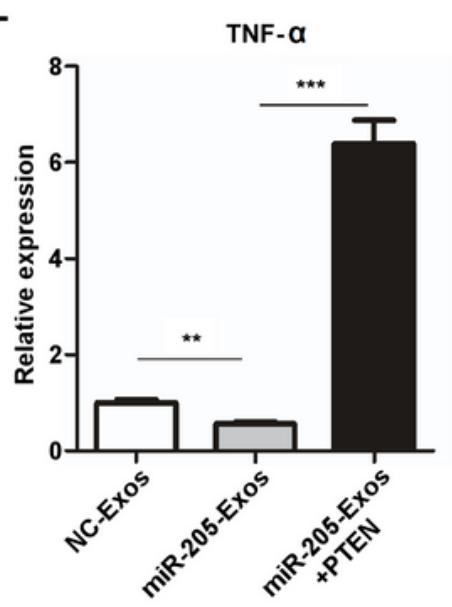

Figure 4 
Exosomal miR-205 regulates M2 macrophage polarization by targeting PTEN. a After treatment with the miR-205 inhibitor, NC or mimic, the miR-205 mRNA levels in macrophages were measured by RT-PCR. $b$ The inhibition of PTEN expression after treatment with the miR-205 inhibitor, NC or mimics was assessed by Western blotting. c Western blotting was used to detect PTEN and a-tubulin in macrophages incubated with NC, miR-205 mimics or miR-205 mimics+PTEN. d Western blotting was used to detect PTEN and atubulin in macrophages incubated with NC-Exos, miR-205-Exos or miR-205-Exos+PTEN. (E-H) The effects of NC-Exos, miR-205-Exos and miR-205-Exos+PTEN on the expression levels of M1-type (IL-1 $\beta$ and TNFa) and M2-type (CD163 and IL-10) markers were analysed by qRT-PCR in macrophages. All experiments were repeated three times, and the results are presented as the mean \pm SEM. Statistical significance was determined by a two-tailed, unpaired Student's t test. ${ }^{\star} \mathrm{P}<0.05,{ }^{* \star} \mathrm{P}<0.01$ and ${ }^{* \star *} \mathrm{P}<0.001$. 
A

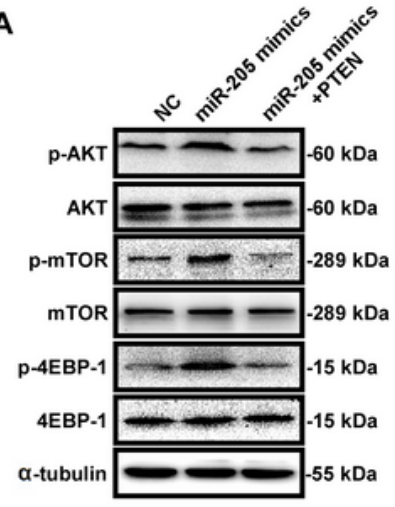

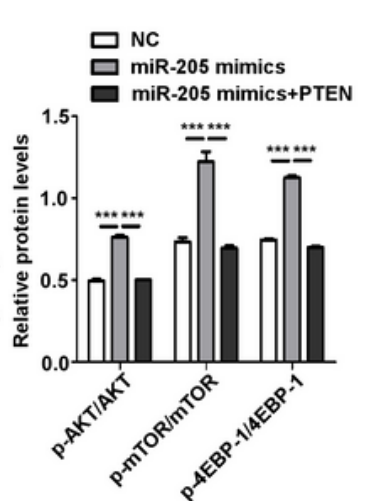

B

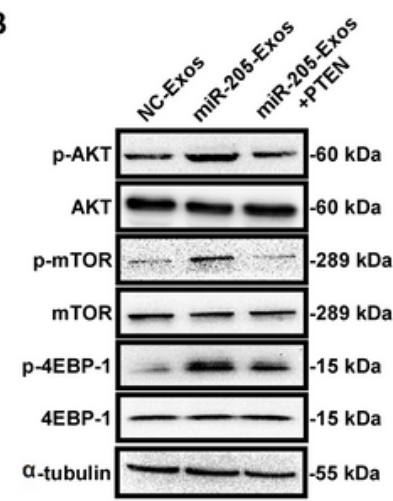

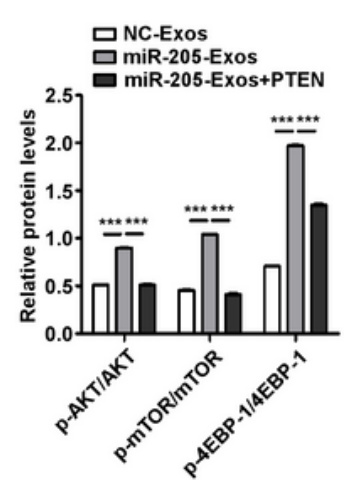

C
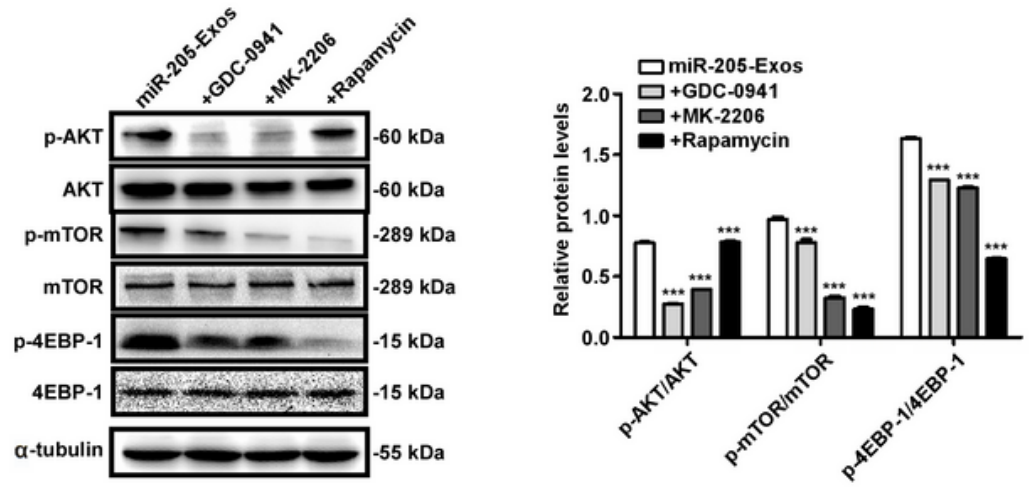

D

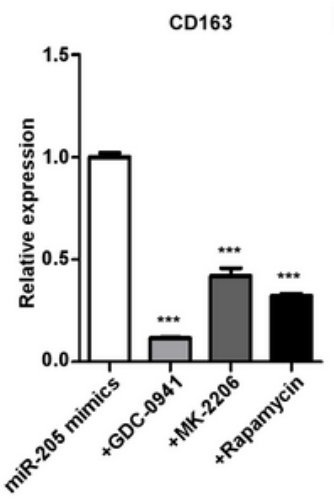

E $\quad \mathrm{LL}-10$

F

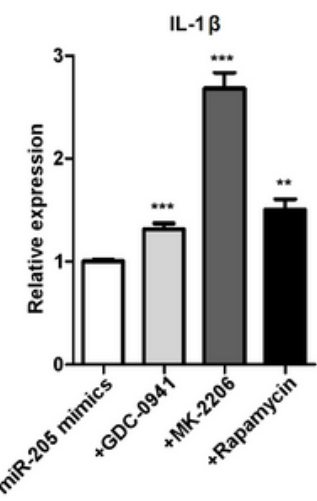

G

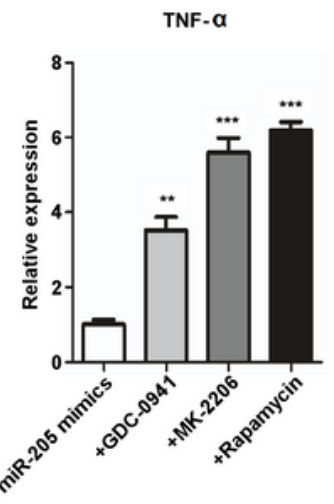

H

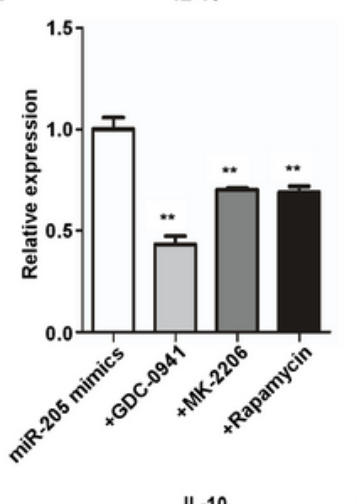

IL-1 $\beta$
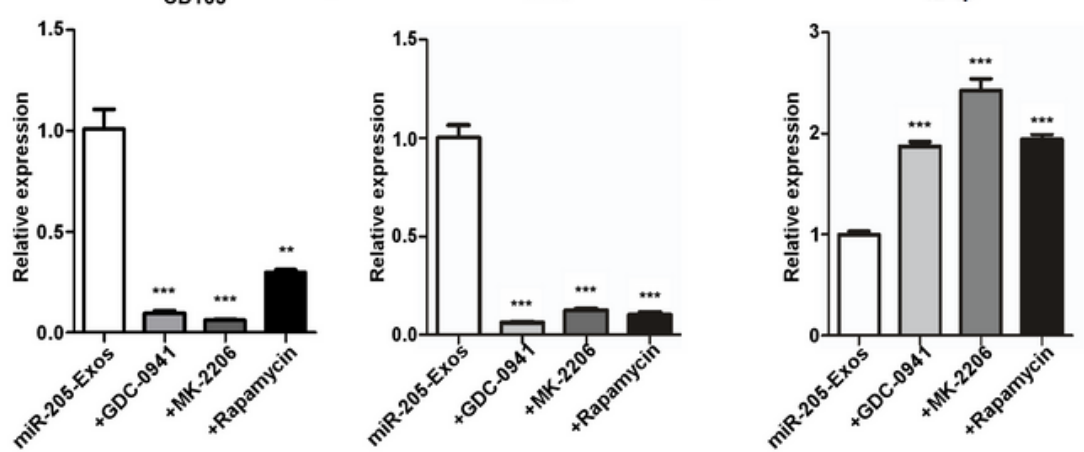

K

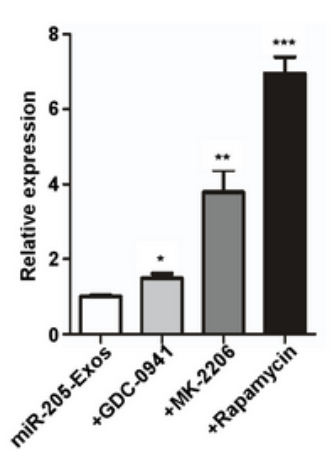

Figure 5

miR-205 regulates macrophage polarization by activating the PTEN/AKT/mTOR signalling pathway. a After transfection with NC, miR-205 mimics or miR-205 mimics+PTEN, p-AKT, AKT, p-mTOR, mTOR, p4EBP-1, 4EBP-1 and a-tubulin in macrophages were detected by Western blotting. $\mathrm{b}$ After incubation with NC-Exos, miR-205-Exos or miR-205-Exos+PTEN, Western blotting was used to detect PTEN, p-AKT, AKT, pmTOR, mTOR, p-4EBP-1, 4EBP-1 and a-tubulin in macrophages. c Western blot analysis shows the effects 
of miR-205-Exos, miR-205-Exos+GDC-0941, miR-205-Exos+MK-2206 and miR-205-Exos+rapamycin on the protein levels of p-AKT, AKT, p-mTOR, mTOR, p-4EBP-1, 4EBP-1 and a-tubulin. d-g After transfection with miR-205 mimics, the effects of GDC-0941, MK-2206 and rapamycin on the expression levels of M1-type (IL-1 $\beta$ and TNF-a) and M2-type (CD163 and IL10) markers were analysed by qRT-PCR. h-k After incubation with miR-205-Exos, the effects of GDC-0941, MK-2206 and rapamycin on M1-type (IL-1 $\beta$ and TNF-a) and M2-type (CD163 and IL10) marker expression levels were analysed by qRT-PCR. All experiments were repeated three times, and the results are presented as the mean $\pm S E M$. Statistical significance was determined by a two-tailed, unpaired Student's $t$ test. ${ }^{*} P<0.05$, ${ }^{*} P<0.01$ and ${ }^{* \star *} P<0.001$. n.s, not significant. 
A
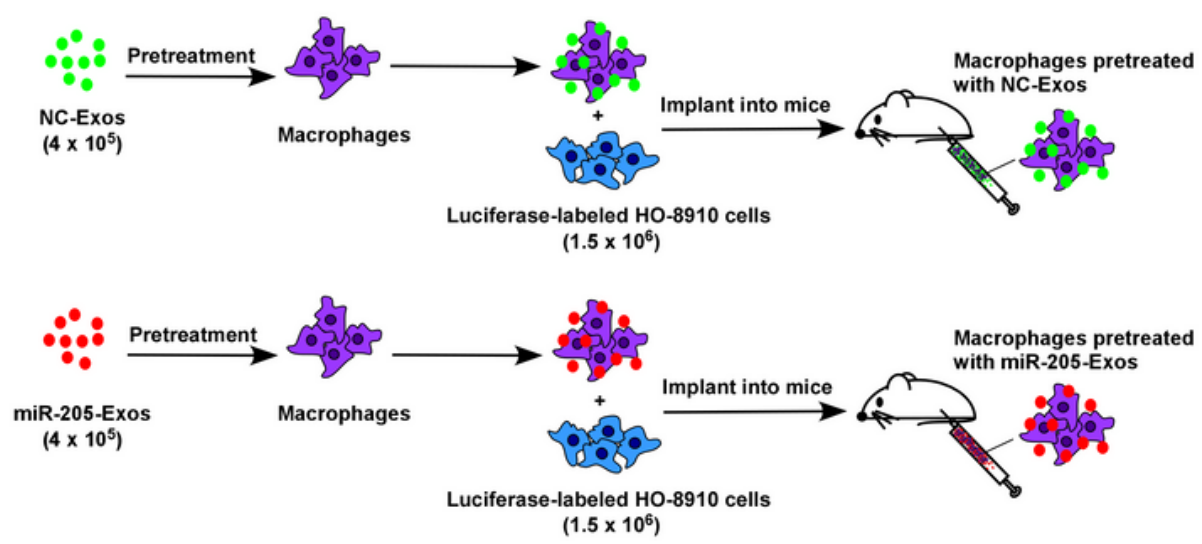

B
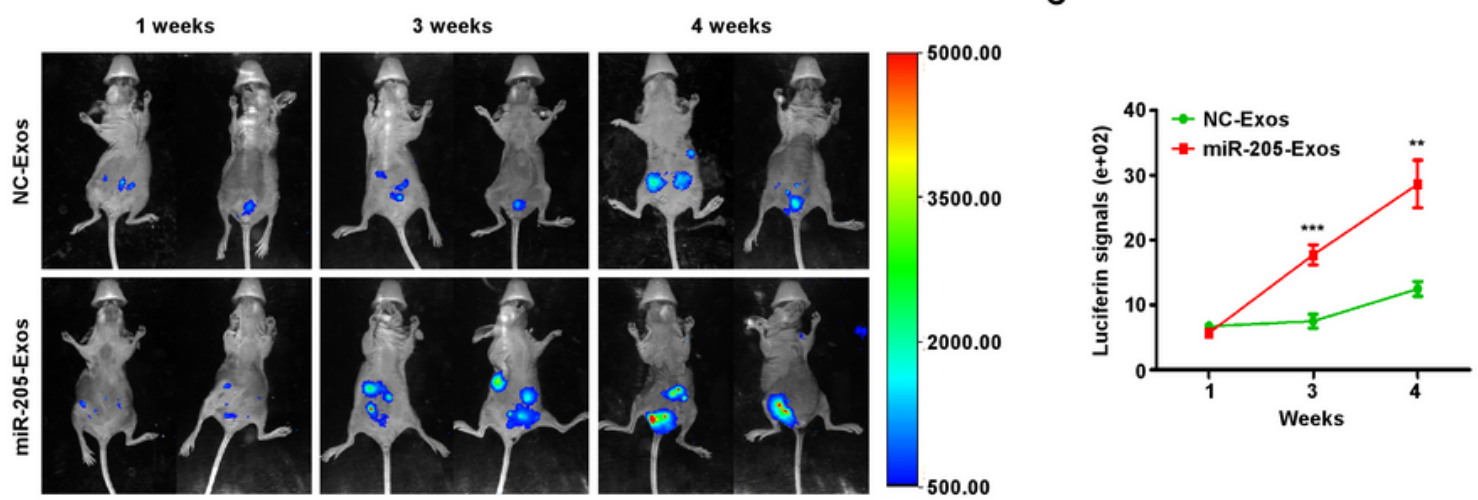

D
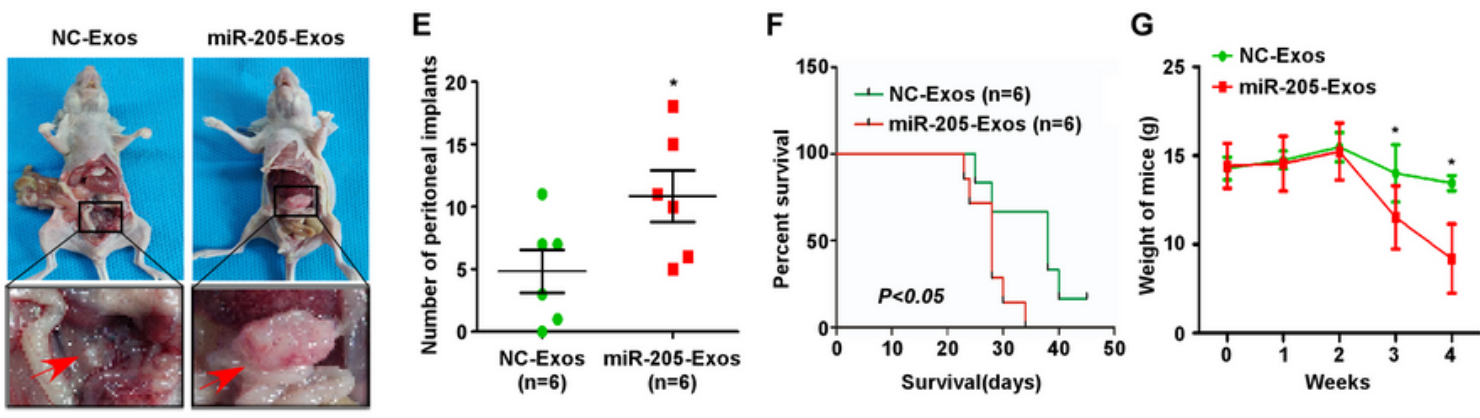

H
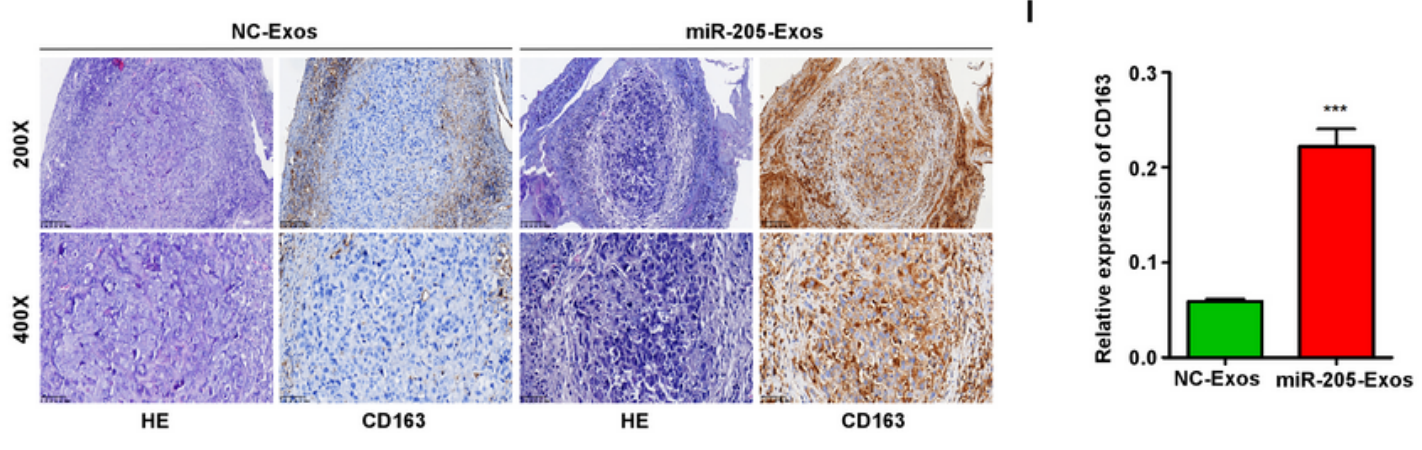

Figure 6

miR-205 promotes OC tumorigenesis in vivo. a Macrophages pretreated with miR-205-Exos or NC-Exos were mixed with luciferase-labelled HO-8910 cells, and then the mixtures were injected into the abdominal cavities of nude mice ( $n=6$ each). Thereafter, macrophages pretreated with exosomes were injected into the abdominal cavities of the mice every 5 days. b Representative images of mice at different time points between 1 week and 4 weeks (from left to right) after injection with the mixtures. After an i.p. injection od 
$100 \mathrm{mg} / \mathrm{kg}$ D-luciferin, the mice were anaesthetized with $2.0 \%$ isofluorane and imaged by the IVIS system every week. Colour bars represent tumour cell intensity from low (blue) to high (red). c Luciferase activities of the peritoneal tumours were measured every week. $d$ Representative images of peritoneal tumours in nude mice that resulted from HO-8910 cells coinjected with macrophages pretreated with miR205-Exos or NC-Exos derived from OC cells. e The number of peritoneal tumour implants in each indicated group. $f$ The survival status of each mouse was recorded daily. Kaplan-Meier analysis was used to analyse the overall survival rates of nude mice in the indicated groups. $g$ The weight of mice was monitored weekly after the mice were injected with pretreated macrophages. $\mathrm{h}$ and i Mouse xenografts were subjected to $\mathrm{HE}$ and IHC staining for CD163, and the relative expression of CD163 was quantitatively assessed. Data are presented as the mean $\pm S E M ; * P<0.05,{ }^{\star} P<<0.01, \star \star \star P<0.001$.

\section{Supplementary Files}

This is a list of supplementary files associated with this preprint. Click to download.

- Scheme.1.tif

- Supplementary.docx 